\title{
Nonlinear Dynamics of Clamped Initial Imperfect Functionally Graded Material Circular Cylindrical Shell Considering the Axisymmetric Mode
}

\author{
Yuxin $\mathrm{Hao}^{1, *}$, Wei $\mathrm{Li}^{1}$, Wei Zhang ${ }^{2}$, Shuangbao $\mathrm{Li}^{3}$ \\ and Minghui $\mathrm{Yao}^{2}$ \\ ${ }^{1}$ College of Mechanical Engineering, Beijing Information Science and Technology \\ University, Beijing 100192, China \\ ${ }^{2}$ College of Mechanical Engineering, Beijing University of Technology, Beijing 100124, \\ China \\ ${ }^{3}$ College of Science, Civil Aviation University of China, Tianjin 300300, China
}

Received 26 January 2017; Accepted (in revised version) 18 May 2017

\begin{abstract}
This paper investigates the dynamic responses of clamped-clamped functionally graded material circular cylindrical shell at both ends with small initial geometric imperfection and subjected to complex loads. The small initial geometric imperfection of the cylindrical shell is characterized with the shape of hyperbolic function. The effects of radial harmonic excitation combined with thermal loads are considered. The classical theory and von-Karman type nonlinear geometric equation are applied to obtain partial differential equation of the functionally gradient material circular cylindrical shell by the Hamilton's principle. The partial differential dynamic equations are truncated by the Galerkin technique, using the modal expansion with the inclusion of axisymmetric and asymmetric modes. The effective material properties vary in the radial direction following a power-law distribution accordance with the volume fractions. The effects of volume fraction indexes, ratios of thickness-radius and lengthradius on the first three dimensionless natural frequencies of the perfect cylindrical shell and its counterpart with imperfection are given. The effects of radial external loads, initial geometric imperfections and volume fraction index on the nonlinear dynamic response of the clamped-clamped FGM circular cylindrical shell are discussed by numerical calculation.
\end{abstract}

AMS subject classifications: 34A34, 34K18

Key words: Vibration, circular cylindrical shell, functionally graded materials, geometric imperfections, clamped-clamped.

*Corresponding author.

Emails: bimhao@163.com (Y.X. Hao), sandyzhang0@yahoo.com (W. Zhang) 


\section{Introduction}

The functionally gradient material (FGM) circular cylindrical shell structures whose material properties vary gradually and smoothly in thickness direction have been found to be widely applied in space vehicles, nuclear plants and so on due to their capacity to withstand high levels of thermal, mechanical and acoustic pressure. Under complicated working conditions these shells may cause a complicated nonlinear dynamics [1].

Recently, the dynamics of FGM circular cylindrical shells have attracted increasing research efforts. Firstly, the focus is put on the studies of natural frequencies of the FGM circular cylindrical shell. Some works dealt with the effect of physical and geometric parameters and edge conditions on the value of natural frequencies for functionally graded material circular cylindrical shell, see [2-5]. Assumed the axisymmetric conditions, Asgari and Akhlaghi [6] presented natural frequencies of thick hollow limited length FGM cylinders, according to 3D equations of elasticity.

Bhangale and Ganesan [7] analyzed the frequency behavior of vibrating nonhomogeneous FGM magneto-electro-elastic finite cylindrical shells with simply supported boundary (SS). The influence of magnetic and piezoelectric on the frequency of structures is evaluated. The free vibration of functionally graded material cylindrical shells was studied by Cao and Wang [8]. There are some holes in the FGM cylindrical shells. The effects of holes shape, number and location on the frequencies were analyzed. Sepiani et al. [9] researched the buckling and free vibration properties of cylindrical shell with static and harmonic excitation along axial direction. The inner of the shell is the FGM layer and the out surface of the circular cylindrical shell is isotropic elastic layer. Both the first order shear deformation theory and the classical shell theory were used in theoretical formulations.

Using the generalized thermo-elasticity theory and second-order shear deformation shell theory, Bahtui and Eslami [10] researched the response of FGM circular cylindrical shell which is subjected to thermal shock load. Considering the effect of thermal and mechanical coupling combined with rotary inertia, Galerkin method and the Laplace transform were used to formulate the problem. $\mathrm{Ng}$ et al. [11] studied the influences of the volume fraction on the parametric response of FGM cylindrical shells under harmonic axial excitation, especially the positions and range of stability.

About the nonlinear dynamics, Mahmoudkhani et al. [12] used Donnell's nonlinear shallow shell theory and multiple scales methods to study the periodic solutions and their stability of simply supported circular cylindrical shells with the primary resonance. The sufficient and necessary conditions for the emergence of companion mode were discussed. Bichetal and Nguyen [13] investigated nonlinear amplitude frequency response and nonlinear dynamic of FGM circular cylindrical shells by using Donnell shell theory. Hao et al. [14] presented the nonlinear dynamics of SS functionally graded material circular cylindrical shell with 1:2 internal resonance between first two modes but without considering the axisymmetric mode. On the condition that primary resonance and 1:2 internal resonance of the system between two modes, $\mathrm{Du}$ and Li [15] dealt with the non- 
linear vibrations of FGM cylindrical shells with simply supported boundaries. Shen [16] studied nonlinear free vibrations of FGM cylindrical shells surrounded by Pasternak foundation. Strozzi and Pellicano [17] focused on the nonlinear vibrations character of FGM circular cylindrical shells by using the Sanders-Koiter theory. Sheng et al. $[18,19]$ dealt with the nonlinear vibration control of laminated FGM cylindrical shells based on the approach of constant gain negative velocity feedback. And also they studied nonlinear vibration of functionally graded material circular cylindrical shell surrounded by an elastic foundation in the case of the primary resonance.

In above studies, most of them focused on FGM cylindrical shells with the SS boundary conditions. The research on the dynamics of clamped-clamped (CC) FGM cylindrical shell at both ends are limited in number. Kadoli and Ganesan [20] analyzed linear free vibration and thermal buckling for FGM cylindrical shells with CC boundary condition at two ends and material properties are temperature dependent. In a uniform temperature field, Zhang et al. [21] analyzed the nonlinear dynamics of functionally graded material circular cylindrical shell with CC boundary. The shell is subjected to the combined external load. Alijani and Amabili [22] reviewed geometric nonlinear forced and free vibrations of shells that were made of traditional or advanced materials. It is presented that most of the studies neglected the effect of axisymmetric modes in closed shells. So a more careful investigation is required in order to illustrate the nonlinear dynamic behavior of thin walled revolving structures.

It is known that small deviations in shape of the shells configuration because of fabrication are inherent in most real structures. These geometric imperfections can affect the dynamic behavior of the shells considerably, see Rafiee et al. [23]. When one considers the effects of the combined external loads, the dynamics of the imperfect cylindrical shell are usually nonlinearity and become very complex. Therefore it is important and necessary to have a good knowledge about the dynamic response of cylindrical shell with imperfection. In the literature available, many papers discussed the buckling behaviors of imperfect FGM cylindrical shells, see [24-30]. And there is only little work dealing with dynamics of the imperfect FGM cylindrical shells.

For the nonlinear continuous dynamics systems of FGM structures, they are infinitedimensional and it is necessary to discretize them into a finite dimension dynamic system. There are some method usually used to discretize them such as multi-symplectic method, modal expansion and so on. The generalized multi-symplectic method proposed by $\mathrm{Hu}$ [31] is a specific finite difference method and the same authors [32-34] applied the generalized multi-symplectic method to solve the vibration of the damping beam. Also, they researched the chaotic motions of the embedded single-walled carbon nanotube by considering the geometrical nonlinearity problems. On the other hand, the solutions for the nonlinear continuous dynamics systems are assumed to be a finite linear combination of chosen function with undetermined coefficients. And the solutions are approximated to the exact solution of the problem [35].

In this paper, a nonlinear dynamic analysis of a slightly initial imperfect FGM clamped-clamped circular cylindrical shell at both ends which is subjected to radial loads 
is presented. The small initial geometric imperfection of the cylindrical shell is assumed to have the same form of shape function with radial deflection. The material properties are varied as a power-law accordance with the volume fractions along the radial direction. According to the classical shell theory and von-Karman geometric nonlinearity, the nonlinear partial differential equation of FGM circular cylindrical shell with initial geometric imperfection is deduced by the Hamilton's principle. The partial differential governing equations are truncated by the Galerkin technique. The modal expansion which includes the axisymmetric and asymmetric modes is used in this process since the axisymmetric modes are important in nonlinear oscillations of shell, see [36,37]. The effects of volume fraction indexes, length-radius and thickness-radius ratios on natural frequencies of the cylindrical shell with imperfection and without imperfection are given. The 4th order Runge-Kutta algorithm is used to study the nonlinear dynamics of this system.

\section{Formulation}

An imperfect FGM circular cylindrical thin shell which the geometrical dimensions are length $L$, thickness $h$ and mid-surface radius $R$, respectively. The FGM circular cylindrical shell is assumed to be made of a mixture of metal and ceramic. Both two end edges of the cylindrical shell are clamped. The external force acting on the shell is along the radial direction. A cylindrical coordinates system $(x, \theta, z)$ is located on the mid-plane, where $x$, $\theta$, and $z$ denote respectively the axial direction, circumferential and radial directions of the circular cylindrical shell. The deformations of mid-surface defined in the cylindrical coordinates are $u, v, w$ in the $x, \theta$, and $z$ directions showed in Fig. 1, respectively. According to the classical shell theory, the displacement field of the imperfect FGM circular cylindrical thin shell can be given as

$$
\begin{aligned}
& u(x, \theta, z, t)=u_{0}(x, \theta, t)-z \frac{\partial w_{0}}{\partial x}, \\
& v(x, \theta, z, t)=v_{0}(x, \theta, t)-z \frac{\partial w_{0}}{R \partial \theta}, \\
& w(x, \theta, z, t)=w_{0}(x, \theta, t)+w^{*}(x, \theta),
\end{aligned}
$$

where $w *$. denotes a known small initial geometric imperfection and $u_{0}, v_{0}$ and $w_{0}$ represent the displacements of a point in the middle plane of the FGM shell.

\subsection{Material properties of FGM}

Here the material properties of the cylindrical shell change from inner surface to outer gradually according to a power law based on volume fractions. The effective temperature dependent material properties $P$, such as mass density $\rho$, Young's modulus $E$, and thermal expansion coefficient $\alpha$ are considered as

$$
P(T)=P_{0}\left(P_{-1} T^{-1}+1+P_{1} T+P_{2} T^{2}+P_{3} T^{3}\right),
$$



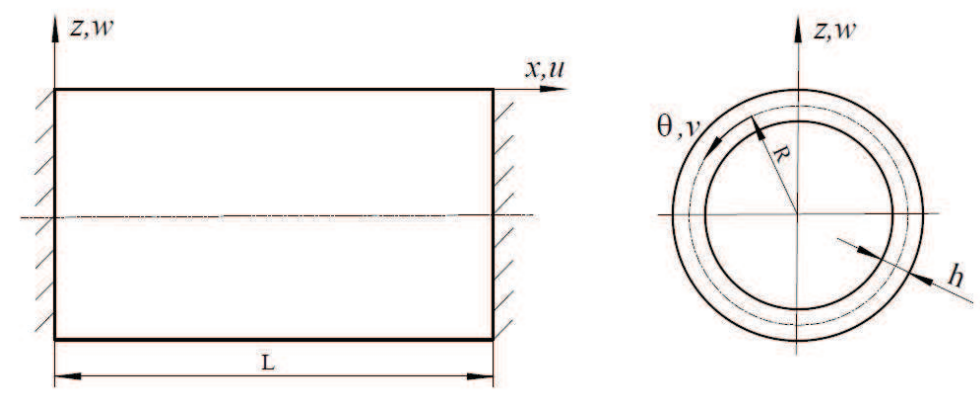

Figure 1: The model of an FGM cylindrical shell and the coordinate system.

where $P_{0}, P_{-1}, P_{1}, P_{2}$ and $P_{3}$ are the coefficients of temperature $T$, see [38].

The volume fraction of ceramic $V_{c}$ can be written in a simple power law as

$$
V_{c}(z)=\left(\frac{2 z+h}{2 h}\right)^{n^{\prime}},
$$

where $n^{\prime}$ defines volume fraction index.

And the volume fraction of metal $V_{m}$ is related by

$$
V_{m}=1-V_{c}
$$

where the subscript $m$ and $c$, respectively represent metal and ceramic. Thus material properties of FGM shell are expressed as

$$
P=P_{c} V_{c}+P_{m} V_{m}
$$

It is supposed that temperature varies along the thickness and we define it by a linear function as

$$
T(z)=T_{m}+\left(T_{c}-T_{m}\right) \eta(z),
$$

where $\eta(z)$ is a polynomial series as follows, see [39]

$$
\begin{aligned}
\eta(z)= & \frac{1}{\mathrm{C}}\left[\left(\frac{z}{h}+\frac{1}{2}\right)-\frac{k_{c m}}{k_{m}(n+1)}\left(\frac{z}{h}+\frac{1}{2}\right)^{n^{\prime}+1}+\frac{k_{c m}^{2}}{k_{m}^{2}(2 n+1)}\left(\frac{z}{h}+\frac{1}{2}\right)^{\left(2 n^{\prime}+1\right)}\right. \\
& \left.-\frac{k_{c m}^{3}}{k_{m}^{3}(3 n+1)}\left(\frac{z}{h}+\frac{1}{2}\right)^{\left(3 n^{\prime}+1\right)}+\frac{k_{c m}^{4}}{k_{m}^{4}(4 n+1)}\left(\frac{z}{h}+\frac{1}{2}\right)^{\left(4 n^{\prime}+1\right)}-\frac{k_{c m}^{5}}{k_{m}^{5}(5 n+1)}\left(\frac{z}{h}+\frac{1}{2}\right)^{\left(5 n^{\prime}+1\right)}\right],
\end{aligned}
$$

with $k_{c m}=k_{c}-k_{m}$, and

$$
C=1-\frac{k_{c m}}{(n+1) k_{m}}+\frac{k_{c m}^{2}}{(2 n+1) k_{m}^{2}}-\frac{k_{c m}^{3}}{(3 n+1) k_{m}^{3}}+\frac{k_{c m}^{4}}{(4 n+1) k_{m}^{4}}-\frac{k_{c m}^{5}}{(5 n+1) k_{m}^{5}},
$$

where $k_{c}$ and $k_{m}$ are the thermal conductivity of ceramic and metal, respectively. 
The small initial geometric imperfection $w^{*}$ in radial direction is considered and can be described as, see [40]

$$
w^{*}=h_{1} \Phi_{m}(x) \cos (\mathrm{n} \theta)+h_{2} \Phi_{m}(x)
$$

where

$$
\begin{aligned}
& \Phi_{m}(x)=\cosh \frac{\lambda_{m} x}{L}-\cos \frac{\lambda_{m} x}{L}-\sigma_{m}\left(\sinh \frac{\lambda_{m} x}{L}-\sin \frac{\lambda_{m} x}{L}\right), \\
& \sigma_{m}=\frac{\cosh \lambda_{m}-\cos \lambda_{m}}{\sinh \lambda_{m}-\sin \lambda_{m}}, \quad \cosh \lambda_{m} \cdot \cos \lambda_{m}=1,
\end{aligned}
$$

where the constant coefficient $h_{1}$ and $h_{2}$ represent the amplitude of the geometrical imperfection.

\subsection{Basic equations}

Based on von-Karman strain-displacement relation and Eq. (2.1), one can be obtained the strain components as follows

$$
\left\{\begin{array}{l}
\varepsilon_{x} \\
\varepsilon_{\theta} \\
\gamma_{\theta z}
\end{array}\right\}=\left\{\begin{array}{c}
\varepsilon_{x}^{(0)} \\
\varepsilon_{\theta}^{(0)} \\
\gamma_{x \theta}^{(0)}
\end{array}\right\}+z\left\{\begin{array}{c}
\varepsilon_{x}^{(1)} \\
\varepsilon_{\theta}^{(1)} \\
\gamma_{x \theta}^{(1)}
\end{array}\right\}
$$

where

$$
\begin{aligned}
& \left\{\begin{array}{c}
\varepsilon_{x}^{(0)} \\
\varepsilon_{\theta}^{(0)} \\
\gamma_{x \theta}^{(0)}
\end{array}\right\}=\left\{\begin{array}{c}
\frac{\partial u_{0}}{\partial x}+\frac{1}{2}\left(\frac{\partial w_{0}}{\partial x}\right)^{2}+\frac{\partial w_{0}}{\partial x} \frac{\partial w^{*}}{\partial x} \\
\frac{1}{R} \frac{\partial v_{0}}{\partial \theta}+\frac{w_{0}}{R}+\frac{1}{2} \frac{1}{R^{2}}\left(\frac{\partial w_{0}}{\partial \theta}\right)^{2}+\frac{1}{R^{2}} \frac{\partial w_{0}}{\partial \theta} \frac{\partial w^{*}}{\partial \theta} \\
\frac{1}{R} \frac{\partial u_{0}}{\partial \theta}+\frac{\partial v_{0}}{\partial x}+\frac{1}{R} \frac{\partial w_{0}}{\partial x} \frac{\partial w_{0}}{\partial \theta}+\frac{1}{R} \frac{\partial w_{0}}{\partial x} \frac{\partial w^{*}}{\partial \theta}+\frac{1}{R} \frac{\partial w_{0}}{\partial \theta} \frac{\partial w^{*}}{\partial x}
\end{array}\right\}, \\
& \left\{\begin{array}{c}
\varepsilon_{x}^{(1)} \\
\varepsilon_{\theta}^{(1)} \\
\gamma_{x \theta}^{(1)}
\end{array}\right\}=\left\{\begin{array}{c}
-\frac{\partial^{2} w_{0}}{\partial x^{2}} \\
-\frac{1}{R^{2}} \frac{\partial^{2} w_{0}}{\partial \theta^{2}} \\
-2 \frac{1}{R} \frac{\partial^{2} w_{0}}{\partial x \partial \theta}
\end{array}\right\} .
\end{aligned}
$$

The constitutive relations including thermal effects are given as

$$
\left.\left\{\begin{array}{l}
\sigma_{x} \\
\sigma_{\theta} \\
\sigma_{x \theta}
\end{array}\right\}=\left[\begin{array}{lll}
Q_{11} & Q_{12} & \\
Q_{12} & Q_{22} & \\
& & Q_{66}
\end{array}\right]\left\{\begin{array}{l}
\varepsilon_{x} \\
\varepsilon_{\theta} \\
\gamma_{x \theta}
\end{array}\right\}-\left\{\begin{array}{l}
\alpha \\
\alpha \\
0
\end{array}\right\} \Delta T\right\},
$$


where

$$
Q_{11}=Q_{22}=\frac{E}{1-v^{2}}, \quad Q_{12}=\frac{v E}{1-v^{2}}, \quad Q_{66}=\frac{E}{2(1+v)} .
$$

The nonlinear dynamic equations for the imperfect FGM circular cylindrical shell under radial dynamic load can be determined by employing the Hamilton's principle

$$
\begin{aligned}
& N_{x x, x}+\frac{1}{R} N_{x \theta, \theta}=I_{0} \ddot{u}_{0}-I_{1} \ddot{w}_{0, x}, \\
& N_{x \theta, x}+\frac{1}{R} N_{\theta \theta, \theta}=I_{0} \ddot{v}_{0}-\frac{1}{R} I_{1} \ddot{w}_{0, \theta}, \\
& M_{x x, x x}+\frac{2}{R} M_{x \theta, x \theta}+\frac{1}{R^{2}} M_{\theta \theta, \theta \theta}+N\left(w_{0}\right)-\frac{1}{R} N_{\theta \theta} \\
& \quad+F-\mu \dot{w}_{0}=I_{0} \ddot{w}_{0}-I_{2}\left(\ddot{w}_{0, x x}+\frac{1}{R^{2}} \ddot{w}_{0, \theta \theta}\right)+I_{1}\left(\ddot{u}_{0, x}+\frac{1}{R} \ddot{v}_{0, \theta}\right),
\end{aligned}
$$

where $F$ is transverse external excitation inertias, $I_{0}, I_{1}$ and $I_{2}$ of the cylindrical shell can be obtained by

$$
I_{i}=\int_{-\frac{h}{2}}^{\frac{h}{2}} z^{i} \rho d z, \quad(i=0,1,2),
$$

and

$$
\begin{aligned}
N\left(w_{0}\right)= & \frac{\partial}{\partial x}\left(N_{x x} w_{0, x}+N_{x x} w_{, x}^{*}+\frac{1}{R} N_{x \theta} w_{0, \theta}+\frac{1}{R} N_{x \theta} w_{, \theta}^{*}\right) \\
& +\frac{1}{R} \frac{\partial}{\partial \theta}\left(\frac{1}{R} N_{x \theta} w_{0, x}+\frac{1}{R} N_{\theta \theta} w_{, \theta}^{*}+\frac{1}{R} N_{\theta \theta} w_{0, \theta}+N_{x \theta} w_{, x}^{*}\right) .
\end{aligned}
$$

All kinds of the stiffness of the FGM cylindrical shell are given by

$$
\left(A_{i j}, B_{i j}, D_{i j}\right)=\int_{-\frac{h}{2}}^{\frac{h}{2}} Q_{i j}\left(1, z, z^{2}\right) d z, \quad(i, j=1,2,6) .
$$

The force and moment resultants including the thermal loads are defined by following integral forms

$$
\begin{aligned}
& \left\{\begin{array}{l}
N_{x x} \\
N_{\theta \theta} \\
N_{x \theta}
\end{array}\right\}=[A],[B]\left\{\begin{array}{l}
\varepsilon^{(0)} \\
\varepsilon^{(1)}
\end{array}\right\}+\left\{\begin{array}{c}
N_{x x}^{T} \\
N_{\theta \theta}^{T} \\
0
\end{array}\right\}, \quad\left\{\begin{array}{l}
M_{x x} \\
M_{\theta \theta} \\
M_{x \theta}
\end{array}\right\}=[B],[D]\left\{\begin{array}{c}
\varepsilon^{(0)} \\
\varepsilon^{(1)}
\end{array}\right\}+\left\{\begin{array}{c}
M_{x x}^{T} \\
M_{\theta \theta}^{T} \\
0
\end{array}\right\}, \\
& \left\{\begin{array}{c}
N_{x x}^{T} \\
N_{\theta \theta}^{T}
\end{array}\right\}=\int_{-\frac{h}{2}}^{\frac{h}{2}}\left[\begin{array}{ll}
Q_{11} & Q_{12} \\
Q_{12} & Q_{22}
\end{array}\right]\left\{\begin{array}{c}
\alpha \\
\alpha
\end{array}\right\} \Delta T d z, \quad\left\{\begin{array}{l}
M_{x x}^{T} \\
M_{\theta \theta}^{T}
\end{array}\right\}=J_{-\frac{h}{2}}^{\frac{h}{2}}\left[\begin{array}{ll}
Q_{11} & Q_{12} \\
Q_{12} & Q_{22}
\end{array}\right]\left\{\begin{array}{c}
\alpha \\
\alpha
\end{array}\right\} \Delta T z d z,
\end{aligned}
$$


Substituting Eqs. (2.16)-(2.19a) into Eq. (2.15a), the nonlinear dynamic models of slightly initial imperfect FGM cylindrical shell can be expanded in form of displacements as

$$
\begin{aligned}
& A_{11}\left(\frac{\partial w_{0}}{\partial x} \frac{\partial^{2} w_{0}}{\partial x^{2}}+\frac{\partial^{2} u_{0}}{\partial x^{2}}\right)+\frac{1}{R} A_{12}\left(\frac{\partial^{2} v_{0}}{\partial x \partial \theta}+\frac{1}{R} \frac{\partial w_{0}}{\partial \theta} \frac{\partial^{2} w_{0}}{\partial x \partial \theta}+\frac{\partial w_{0}}{\partial x}\right)+\frac{1}{R^{2}} A_{66}\left(\frac{\partial^{2} u_{0}}{\partial \theta^{2}}+R \frac{\partial^{2} v_{0}}{\partial x \partial \theta}\right. \\
& \left.+\frac{\partial w_{0}}{\partial \theta} \frac{\partial^{2} w_{0}}{\partial x \partial \theta}+\frac{\partial w_{0}}{\partial x} \frac{\partial^{2} w_{0}}{\partial \theta^{2}}\right)-\frac{2}{R^{2}} B_{66} \frac{\partial^{3} w_{0}}{\partial x \partial \theta^{2}}-\frac{1}{R^{2}} B_{12} \frac{\partial^{3} w_{0}}{\partial x \partial \theta^{2}}-B_{11} \frac{\partial^{3} w_{0}}{\partial x^{3}}+N_{x, x}^{T} \\
& +A_{11}\left(\frac{\partial w_{0}}{\partial x} \frac{\partial^{2} w^{*}}{\partial x^{2}}+\frac{\partial^{2} w_{0}}{\partial x^{2}} \frac{\partial w^{*}}{\partial x}\right)+A_{12}\left(\frac{1}{R^{2}} \frac{\partial w^{*}}{\partial \theta} \frac{\partial^{2} w_{0}}{\partial x \partial \theta}+\frac{1}{R^{2}} \frac{\partial w_{0}}{\partial \theta} \frac{\partial^{2} w^{*}}{\partial x \partial \theta}\right) \\
& +\frac{1}{R^{2}} A_{66}\left(\frac{\partial w^{*}}{\partial \theta} \frac{\partial^{2} w_{0}}{\partial x \partial \theta}+\frac{\partial w_{0}}{\partial x} \frac{\partial^{2} w^{*}}{\partial \theta^{2}}+\frac{\partial^{2} w_{0}}{\partial \theta^{2}} \frac{\partial w^{*}}{\partial x}+\frac{\partial w_{0}}{\partial \theta} \frac{\partial^{2} w^{*}}{\partial x \partial \theta}\right)=I_{0} \ddot{u}_{0}-I_{1} \ddot{w}_{0, x}, \\
& \frac{1}{R^{2}} A_{12}\left(\frac{\partial w_{0}}{\partial x} \frac{\partial^{2} w_{0}}{\partial x \partial \theta}+\frac{\partial^{2} u_{0}}{\partial x \partial \theta}\right)+\frac{1}{R^{3}} A_{22}\left(R \frac{\partial^{2} v_{0}}{\partial \theta^{2}}+\frac{\partial w_{0}}{\partial \theta} \frac{\partial^{2} w_{0}}{\partial \theta^{2}}+R \frac{\partial w_{0}}{\partial \theta}\right)+\frac{1}{R} A_{66}\left(R \frac{\partial^{2} v_{0}}{\partial x^{2}}\right. \\
& \left.+\frac{\partial^{2} u_{0}}{\partial x \partial \theta}+\frac{\partial w_{0}}{\partial x} \frac{\partial^{2} w_{0}}{\partial x \partial \theta}+\frac{\partial w_{0}}{\partial \theta} \frac{\partial^{2} w_{0}}{\partial x^{2}}\right)-2 \frac{1}{R} B_{66} \frac{\partial^{3} w_{0}}{\partial x^{2} \partial \theta}-\frac{1}{R^{3}} B_{22} \frac{\partial^{3} w_{0}}{\partial \theta^{3}}-\frac{1}{R} B_{12} \frac{\partial^{3} w_{0}}{\partial x^{2} \partial \theta}+\frac{1}{R} N_{\theta, \theta}^{T} \\
& +\frac{1}{R^{3}} A_{22}\left(\frac{\partial w^{*}}{\partial \theta} \frac{\partial^{2} w_{0}}{\partial \theta^{2}}+\frac{\partial w_{0}}{\partial \theta} \frac{\partial^{2} w^{*}}{\partial \theta^{2}}\right)+\frac{1}{R^{2}} A_{12}\left(\frac{\partial^{2} w_{0}}{\partial x \partial \theta} \frac{\partial w^{*}}{\partial x}+\frac{\partial w_{0}}{\partial x} \frac{\partial^{2} w^{*}}{\partial x \partial \theta}\right) \\
& +\frac{1}{R} A_{66}\left(\frac{\partial w_{0}}{\partial x} \frac{\partial^{2} w^{*}}{\partial x \partial \theta}+\frac{\partial w^{*}}{\partial \theta} \frac{\partial^{2} w_{0}}{\partial x^{2}}+\frac{\partial^{2} w_{0}}{\partial x \partial \theta} \frac{\partial w^{*}}{\partial x}+\frac{\partial w_{0}}{\partial \theta} \frac{\partial^{2} w^{*}}{\partial x^{2}}\right)=I_{0} \ddot{v}_{0}-\frac{1}{R} I_{1} \ddot{w}_{0, \theta}, \\
& \frac{2}{R^{2}} B_{66}\left(\frac{\partial^{2} w_{0}}{\partial x^{2}} \frac{\partial^{2} w_{0}}{\partial \theta^{2}}+\frac{\partial^{3} u_{0}}{\partial x \partial \theta^{2}}-\frac{\partial^{3} w_{0}}{\partial x \partial \theta^{2}}\right)+\frac{1}{R^{2}} A_{66}\left(4 \frac{\partial w_{0}}{\partial x} \frac{\partial w_{0}}{\partial \theta} \frac{\partial^{2} w_{0}}{\partial x \partial \theta}+\frac{\partial w_{0}}{\partial x} \frac{\partial^{2} u_{0}}{\partial \theta^{2}}+2 \frac{\partial^{2} w_{0}}{\partial x \partial \theta} \frac{\partial u_{0}}{\partial \theta}\right. \\
& \left.+\frac{\partial w_{0}}{\partial \theta} \frac{\partial^{2} u_{0}}{\partial x \partial \theta}+\frac{\partial^{2} w_{0}}{\partial \theta^{2}} \frac{\partial^{2} w_{0}}{\partial x^{2}}+\frac{\partial^{2} w_{0}}{\partial x^{2}} \frac{\partial^{2} w_{0}}{\partial \theta^{2}}+2 R \frac{\partial^{2} w_{0}}{\partial x \partial \theta} \frac{\partial v_{0}}{\partial x}+R \frac{\partial w_{0}}{\partial \theta} \frac{\partial^{2} v_{0}}{\partial x^{2}}+R \frac{\partial w_{0}}{\partial x} \frac{\partial^{2} v_{0}}{\partial x \partial \theta}\right) \\
& +\frac{1}{R^{2}} B_{12}\left(\frac{\partial^{3} u_{0}}{\partial x \partial \theta^{2}}+2 \frac{\partial^{3} w_{0}}{\partial x \partial \theta^{2}}+R \frac{\partial^{3} v_{0}}{\partial x^{2} \partial \theta}-2 \frac{\partial^{2} w_{0}}{\partial x^{2}} \frac{\partial^{2} w_{0}}{\partial \theta^{2}}+2 R \frac{\partial^{2} w_{0}}{\partial x^{2}}\right)+\frac{1}{R^{3}} B_{22}\left(2 R \frac{\partial^{2} w_{0}}{\partial \theta^{2}}\right. \\
& \left.+R \frac{\partial^{3} v_{0}}{\partial \theta^{3}}\right)+\frac{1}{R^{4}} A_{22}\left(R w_{0} \frac{\partial^{2} w_{0}}{\partial \theta^{2}}+R \frac{\partial^{2} w_{0}}{\partial \theta^{2}} \frac{\partial v_{0}}{\partial \theta}+R \frac{\partial w_{0}}{\partial \theta} \frac{\partial^{2} v_{0}}{\partial \theta^{2}}+\frac{3}{2} \frac{\partial^{2} w_{0}}{\partial \theta^{2}} \frac{\partial^{2} w_{0}}{\partial \theta^{2}}+\frac{1}{2} R \frac{\partial^{2} w_{0}}{\partial \theta^{2}}\right. \\
& \left.+R^{2} \frac{\partial v_{0}}{\partial \theta}-R^{2} w_{0}\right)+A_{11}\left(\frac{\partial w_{0}}{\partial x} \frac{\partial^{2} u_{0}}{\partial x^{2}}+\frac{3}{2} \frac{\partial^{2} w_{0}}{\partial x^{2}} \frac{\partial^{2} w_{0}}{\partial x^{2}}+\frac{\partial^{2} w_{0}}{\partial x^{2}} \frac{\partial u_{0}}{\partial x}\right)+B_{11} \frac{\partial^{3} u_{0}}{\partial x^{3}}+\frac{\partial^{3} v_{0}}{\partial x^{2} \partial \theta} \\
& +\frac{1}{R^{3}} A_{12}\left(\frac{\partial w_{0}}{\partial \theta} \frac{\partial w_{0}}{\partial x} \frac{\partial^{2} w_{0}}{\partial x \partial \theta}+\frac{\partial w_{0}}{\partial \theta} \frac{\partial^{2} u_{0}}{\partial x \partial \theta}+\frac{\partial^{2} w_{0}}{\partial \theta^{2}} \frac{\partial u_{0}}{\partial x}\right)+\frac{1}{R^{2}} A_{12} \frac{\partial^{2} w_{0}}{\partial x^{2}} \frac{\partial^{2} w_{0}}{\partial \theta^{2}}+\frac{1}{R} A_{12}\left(-\frac{\partial u_{0}}{\partial x}+\frac{1}{2} \frac{\partial^{2} w_{0}}{\partial x^{2}}\right. \\
& \left.+\frac{\partial w_{0}}{\partial x} \frac{\partial^{2} v_{0}}{\partial x \partial \theta}+\frac{\partial^{2} w_{0}}{\partial x^{2}} \frac{\partial v_{0}}{\partial \theta}+w_{0} \frac{\partial^{2} w_{0}}{\partial x^{2}}\right)-\frac{2}{R^{2}} D_{12} \frac{\partial^{4} w_{0}}{\partial x^{2} \partial \theta^{2}}-\frac{1}{R^{4}} D_{22} \frac{\partial^{4} w_{0}}{\partial \theta^{4}}-D_{11} \frac{\partial^{4} w_{0}}{\partial x^{4}}-\frac{4}{R^{2}} D_{66} \frac{\partial^{4} w_{0}}{\partial x^{2} \partial \theta^{2}} \\
& +\frac{1}{R^{2}} \frac{\partial^{2} w_{0}}{\partial \theta^{2}} N_{\theta}^{T}+\frac{1}{R^{2}} M_{\theta, \theta}^{T}+\frac{1}{R^{2}} \frac{\partial w_{0}}{\partial \theta} N_{\theta, \theta}^{T}+M_{x, x x}^{T}-\frac{1}{R} N_{\theta}^{T}+\frac{\partial w_{0}}{\partial x} N_{x, x}^{T}+\frac{\partial^{2} w_{0}}{\partial x^{2}} N_{x}^{T} \\
& +\frac{2}{R^{2}} B_{66}\left(\frac{\partial^{2} w_{0}}{\partial x^{2}} \frac{\partial^{2} w^{*}}{\partial \theta^{2}}+\frac{\partial w_{0}}{\partial \theta} \frac{\partial^{3} w^{*}}{\partial x^{2} \partial \theta}+\frac{\partial w_{0}}{\partial x} \frac{\partial^{3} w^{*}}{\partial x \partial \theta^{2}}+\frac{\partial^{2} w_{0}}{\partial \theta^{2}} \frac{\partial^{2} w^{*}}{\partial x^{2}}\right)+\frac{1}{R^{2}} A_{66}\left(4 \frac{\partial w_{0}}{\partial x} \frac{\partial^{2} w_{0}}{\partial x \partial \theta} \frac{\partial w^{*}}{\partial \theta}\right. \\
& +4 \frac{\partial w_{0}}{\partial x} \frac{\partial w_{0}}{\partial \theta} \frac{\partial^{2} w^{*}}{\partial x \partial \theta}+2 \frac{\partial^{2} w_{0}}{\partial x^{2}} \frac{\partial w_{0}}{\partial \theta} \frac{\partial w^{*}}{\partial \theta}+\frac{\partial^{2} w^{*}}{\partial \theta^{2}} \frac{\partial w_{0}}{\partial x} \frac{\partial w^{*}}{\partial x}+4 \frac{\partial w^{*}}{\partial x} \frac{\partial w_{0}}{\partial \theta} \frac{\partial^{2} w_{0}}{\partial x \partial \theta}+2 \frac{\partial w^{*}}{\partial x} \frac{\partial^{2} w_{0}}{\partial x \partial \theta} \frac{\partial w^{*}}{\partial \theta} \\
& +3 \frac{\partial w^{*}}{\partial x} \frac{\partial w_{0}}{\partial \theta} \frac{\partial^{2} w^{*}}{\partial x \partial \theta}+2 \frac{\partial^{2} w_{0}}{\partial \theta^{2}} \frac{\partial w_{0}}{\partial x} \frac{\partial w^{*}}{\partial x}+3 \frac{\partial w^{*}}{\partial \theta} \frac{\partial w_{0}}{\partial x} \frac{\partial^{2} w^{*}}{\partial x \partial \theta}+\frac{\partial w^{*}}{\partial \theta} \frac{\partial^{2} u_{0}}{\partial x \partial \theta}+\frac{\partial^{2} w_{0}}{\partial x^{2}} \frac{\partial^{2} w^{*}}{\partial \theta^{2}}+\frac{\partial w^{*}}{\partial x} \frac{\partial^{2} u_{0}}{\partial \theta^{2}} \\
& +\frac{\partial^{2} w^{*}}{\partial x^{2}} \frac{\partial w_{0}}{\partial \theta} \frac{\partial w^{*}}{\partial \theta}+\frac{\partial^{2} w_{0}}{\partial \theta^{2}} \frac{\partial^{2} w^{*}}{\partial x^{2}}+2 \frac{\partial^{2} w^{*}}{\partial x \partial \theta} \frac{\partial u_{0}}{\partial \theta}+\frac{\partial^{2} w_{0}}{\partial \theta^{2}} \frac{\partial^{2} w^{*}}{\partial x^{2}}+\frac{\partial^{2} w_{0}}{\partial x^{2}} \frac{\partial^{2} w^{*}}{\partial \theta^{2}} R \frac{\partial w^{*}}{\partial \theta} \frac{\partial^{2} v_{0}}{\partial x^{2}}+2 R \frac{\partial^{2} w^{*}}{\partial x \partial \theta} \frac{\partial v_{0}}{\partial x}
\end{aligned}
$$




$$
\begin{aligned}
& \left.+R \frac{\partial w^{*}}{\partial x} \frac{\partial^{2} v_{0}}{\partial x \partial \theta}\right)+B_{11}\left(\frac{\partial w_{0}}{\partial x} \frac{\partial^{3} w^{*}}{\partial x^{3}}+\frac{\partial^{2} w_{0}}{\partial x^{2}} \frac{\partial^{2} w^{*}}{\partial x^{2}}\right)+\frac{1}{R^{4}} B_{22}\left(\frac{\partial^{2} w_{0}}{\partial \theta^{2}} \frac{\partial^{2} w^{*}}{\partial \theta^{2}}+\frac{\partial w_{0}}{\partial \theta} \frac{\partial^{3} w^{*}}{\partial \theta^{3}}\right) \\
& +\frac{1}{R^{2}} B_{12}\left(4 \frac{\partial^{2} w_{0}}{\partial x \partial \theta} \frac{\partial^{2} w^{*}}{\partial x \partial \theta}+\frac{\partial w_{0}}{\partial \theta} \frac{\partial^{3} w^{*}}{\partial x^{2} \partial \theta}+\frac{\partial w_{0}}{\partial x} \frac{\partial^{3} w^{*}}{\partial x \partial \theta^{2}}-\frac{\partial^{2} w_{0}}{\partial x^{2}} \frac{\partial^{2} w^{*}}{\partial \theta^{2}}-\frac{\partial^{2} w_{0}}{\partial \theta^{2}} \frac{\partial^{2} w^{*}}{\partial x^{2}}\right) \\
& +\frac{1}{R^{4}} A_{22}\left(R w_{0} \frac{\partial^{2} w^{*}}{\partial \theta^{2}}+\frac{3}{2} \frac{\partial^{2} w_{0}}{\partial \theta^{2}} \frac{\partial^{2} w^{*}}{\partial \theta^{2}}+3 \frac{\partial w_{0}}{\partial \theta} \frac{\partial^{2} w_{0}}{\partial \theta^{2}} \frac{\partial w^{*}}{\partial \theta}+2 \frac{\partial^{2} w^{*}}{\partial \theta^{2}} \frac{\partial w_{0}}{\partial \theta} \frac{\partial w^{*}}{\partial \theta}+R \frac{\partial w^{*}}{\partial \theta} \frac{\partial^{2} v_{0}}{\partial \theta^{2}}\right. \\
& \left.+\frac{\partial^{2} w^{*}}{\partial \theta^{2}} \frac{\partial^{2} w_{0}}{\partial \theta^{2}}+R \frac{\partial^{2} w^{*}}{\partial \theta^{2}} \frac{\partial v_{0}}{\partial \theta}\right)+A_{11}\left(2 \frac{\partial w_{0}}{\partial x} \frac{\partial w^{*}}{\partial x} \frac{\partial^{2} w^{*}}{\partial x^{2}}+\frac{3}{2} \frac{\partial^{2} w_{0}}{\partial x^{2}} \frac{\partial^{2} w^{*}}{\partial x^{2}}+\frac{\partial^{2} w_{0}}{\partial x^{2}} \frac{\partial^{2} w^{*}}{\partial x^{2}}+\frac{\partial w^{*}}{\partial x} \frac{\partial^{2} u_{0}}{\partial x^{2}}\right. \\
& \left.+\frac{\partial^{2} w^{*}}{\partial x^{2}} \frac{\partial u_{0}}{\partial x}+3 \frac{\partial w_{0}}{\partial x} \frac{\partial^{2} w_{0}}{\partial x^{2}} \frac{\partial w^{*}}{\partial x}\right)+\frac{1}{R^{3}} A_{12}\left(\frac{\partial w_{0}}{\partial \theta} \frac{\partial^{2} w_{0}}{\partial x \partial \theta} \frac{\partial w^{*}}{\partial x}+\frac{\partial w_{0}}{\partial \theta} \frac{\partial w_{0}}{\partial x} \frac{\partial^{2} w^{*}}{\partial x \partial \theta}+\frac{\partial w^{*}}{\partial \theta} \frac{\partial w_{0}}{\partial x} \frac{\partial^{2} w_{0}}{\partial x \partial \theta}\right. \\
& \left.+\frac{\partial w^{*}}{\partial \theta} \frac{\partial w^{*}}{\partial x} \frac{\partial^{2} w_{0}}{\partial x \partial \theta}+\frac{\partial w^{*}}{\partial \theta} \frac{\partial w_{0}}{\partial x} \frac{\partial^{2} w^{*}}{\partial x \partial \theta}+\frac{\partial w^{*}}{\partial \theta} \frac{\partial^{2} u_{0}}{\partial x \partial \theta}\right)+\frac{1}{R^{2}} A_{12}\left(\frac{\partial w_{0}}{\partial \theta} \frac{\partial w_{0}}{\partial x} \frac{\partial^{2} w_{0}}{\partial x \partial \theta}+\frac{\partial w_{0}}{\partial x} \frac{\partial^{2} w_{0}}{\partial x \partial \theta} \frac{\partial w^{*}}{\partial \theta}\right. \\
& +\frac{\partial w_{0}}{\partial x} \frac{\partial w_{0}}{\partial \theta} \frac{\partial^{2} w^{*}}{\partial x \partial \theta}+\frac{\partial w^{*}}{\partial \theta} \frac{\partial w_{0}}{\partial \theta} \frac{\partial^{2} w_{0}}{\partial x^{2}}+\frac{\partial^{2} w^{*}}{\partial \theta^{2}} \frac{\partial w_{0}}{\partial x} \frac{\partial w^{*}}{\partial x}+\frac{\partial w^{*}}{\partial x} \frac{\partial w_{0}}{\partial \theta} \frac{\partial^{2} w_{0}}{\partial x \partial \theta}+\frac{\partial w^{*}}{\partial x} \frac{\partial^{2} w_{0}}{\partial x \partial \theta} \frac{\partial w^{*}}{\partial \theta} \\
& \left.+\frac{\partial w^{*}}{\partial x} \frac{\partial w_{0}}{\partial \theta} \frac{\partial^{2} w^{*}}{\partial x \partial \theta}+\frac{\partial^{2} w^{*}}{\partial x^{2}} \frac{\partial w_{0}}{\partial \theta} \frac{\partial w^{*}}{\partial \theta}+\frac{\partial w_{0}}{\partial x} \frac{\partial^{2} w_{0}}{\partial \theta^{2}} \frac{\partial w^{*}}{\partial x}+\frac{\partial^{2} w^{*}}{\partial \theta^{2}} \frac{\partial u_{0}}{\partial x}\right)+\frac{1}{2 R^{2}} A_{12}\left(\frac{\partial^{2} w_{0}}{\partial \theta^{2}} \frac{\partial^{2} w^{*}}{\partial x^{2}}\right. \\
& \left.+\frac{\partial^{2} w_{0}}{\partial x^{2}} \frac{\partial^{2} w^{*}}{\partial \theta^{2}}\right)+\frac{1}{R} A_{12}\left(\frac{\partial w^{*}}{\partial x} \frac{\partial^{2} v_{0}}{\partial x \partial \theta}+\frac{\partial^{2} w^{*}}{\partial x^{2}} \frac{\partial v_{0}}{\partial \theta}+w_{0} \frac{\partial^{2} w^{*}}{\partial x^{2}}\right)+\frac{1}{R^{2}} \frac{\partial w^{*}}{\partial \theta} N_{\theta, \theta}^{T}+\frac{\partial w^{*}}{\partial x} N_{x, x}^{T} \\
& +\frac{1}{R^{2}} \frac{\partial^{2} w^{*}}{\partial \theta^{2}} N_{\theta}^{T}+\frac{\partial^{2} w^{*}}{\partial x^{2}} N_{x}^{T}+F-\mu \dot{w}_{0}=I_{0} \ddot{w}_{0}-I_{2}\left(\ddot{w}_{0, x x}+\frac{1}{R^{2}} \ddot{w}_{0, \theta \theta}\right)+I_{1}\left(\ddot{u}_{0, x}+\frac{1}{R} \ddot{v}_{0, \theta}\right) \text {. }
\end{aligned}
$$

Assuming the FGM circular cylindrical shell is clamped-clamped at two ends, which are prescribed in the following form

$$
x=0, \quad x=L: u_{0}=w_{0}=v_{0}=0, \quad \frac{\partial w_{0}}{\partial x}=0 .
$$

\subsection{Solution expansion}

To analyze this nonlinear continuous dynamics system of FGM circular cylindrical shell with small initial geometric imperfection, it is necessary to discretize them into a finite dimension dynamic system by using the modal expansion. For a circular cylindrical shell, the effects of axisymmetric modes should be considered [41-44]. Generally it is difficult for us to take all the series terms when the nonlinear dynamics of the system are analyzed. The approach used commonly is taking a few terms in nonlinear motion governing equations based on the series form that the external excitation is expanded [35]. The research results have shown that the vibration mode which is related to external excitation and the corresponding axisymmetric mode with twice as much as the number of half waves in the axial direction should be retained [15].

The expansions of $u_{0}, v_{0}$ and $w_{0}$, which satisfy clamped-clamped boundary conditions are given as follows, see [35]

$$
u_{0}=\sum_{m=1}^{M} \sum_{n=1}^{N} U_{m n}(t) \frac{d \Phi_{m}(x)}{d x} \cos (\mathrm{n} \theta)+\sum_{m=1}^{M} 2 U_{m 0}(t) \frac{d \Phi_{m}(x)}{d x}
$$




$$
\begin{aligned}
& v_{0}=\sum_{m=1}^{M} \sum_{n=1}^{N} V_{m n}(t) \Phi_{m}(x) \sin (\mathrm{n} \theta), \\
& w_{0}=\sum_{m=1}^{M} \sum_{n=1}^{N} W_{m n}(t) \Phi_{m}(x) \cos (\mathrm{n} \theta)+\sum_{m=1}^{M} 2 W_{m 0}(t) \Phi_{m}(x),
\end{aligned}
$$

where $U_{m n}(t), V_{m n}(t)$ and $W_{m n}(t)$ are time functions, $m$ is the number of axial half waves and $n$ denotes circumferential waves in above equations, see Amabili [45]. The terms

$$
\sum_{m=1}^{M} 2 U_{m 0}(t) \frac{d \Phi_{m}(x)}{d x} \text { and } \sum_{m=1}^{M} 2 W_{m 0}(t) \Phi_{m}(x)
$$

represent axisymmetric mode.

The transverse external excitation can be described as

$$
F=\sum_{m=1}^{M} \sum_{n=1}^{N} f_{m n}(t) \Phi_{m}(x) \cos (\mathrm{n} \theta)+\sum_{m=1}^{M} 2 f_{m 0}(t) \Phi_{m}(x),
$$

where $f_{m n}$ and $f_{m 0}$ are the force amplitude in accordance with the modes of lateral deflection.

Following the results of [46,47], the inertia terms of $u_{0}$ and $v_{0}$ in Eq. (2.20) can be omitted. Based on Galerkin integration procedure [48], a set of nonlinear motion governing equations in the form of radial displacements with two basic vibration modes and one axisymmetric mode relevant to them are obtained as

$$
\ddot{W}_{m}+\mu_{m} \dot{W}_{m}+\sum_{i=1}^{3} C_{m i} W_{i}+\sum_{i=1}^{3} \sum_{j=1}^{3} \sum_{l=1}^{3} \zeta_{m i j} W_{i} W_{j} W_{l}+\xi=\lambda_{m} f \cos (\Omega t), \quad m=1,2,3,
$$

where $\ddot{W}$ and $\dot{W}$ represent the second-order and first-order differentiation with respect to time $t$, respectively. $W_{m}$ denotes the amplitude of every mode, $\mu_{m}$ represents the effect of damping. The coefficients $C_{m i}$ are the linear stiffness terms caused by structure, $\zeta_{m i j}$ are the nonlinear stiffness coefficients associated with quadratic and cubic terms, $\xi$ is the constant matrix caused by the geometric imperfections of the cylindrical shell. The coefficients $\lambda_{m}$ in the right hand are obtained by the Galerkin method. With the aid of computerized symbolic manipulation technique the linear and nonlinear stiffness terms are obtained.

\section{Numerical results}

A numerical analysis is performed by considering the fourth order Runge-Kutta method on an imperfect FGM cylindrical shell. The validation of numerical calculation programs used in present was demonstrated in our previous works done by Yang and Hao [49] since these two dynamic systems have the similar expressions. For example, the quadratic 
Table 1: The temperature-dependent coefficients of SUS304/SI ${ }_{3} \mathrm{~N}_{4}$.

\begin{tabular}{|c|c|c|c|c|c|}
\hline Material & $P_{0}$ & $P_{-1}$ & $P_{1}$ & $P_{2}$ & $P_{3}$ \\
\hline Modulus of elasticity (in Pa) & & & & & \\
\hline SUS304 & $210.04 \times 10^{9}$ & 0 & $3.079 \times 10^{-4}$ & $-6.534 \times 10^{-7}$ & 0 \\
\hline $\mathrm{SI}_{3} \mathrm{~N}_{4}$ & $348.43 \times 10^{9}$ & 0 & $-3.070 \times 10^{-4}$ & $2.160 \times 10^{-7}$ & $-8.946 \times 10^{-11}$ \\
\hline Mass density (in $\mathrm{kg} / \mathrm{m}^{3}$ ) & & & & & \\
\hline SUS304 & 8166 & 0 & 0 & 0 & 0 \\
\hline $\mathrm{SI}_{3} \mathrm{~N}_{4}$ & 2370 & 0 & 0 & 0 & 0 \\
\hline Thermal expansion (in $1 / \mathrm{K}$ ) & & & & & \\
\hline SUS304 & $12.330 \times 10^{-6}$ & 0 & $8.086 \times 10^{-4}$ & 0 & 0 \\
\hline $\mathrm{SI}_{3} \mathrm{~N}_{4}$ & $5.8723 \times 10^{-6}$ & 0 & $9.095 \times 10^{-4}$ & 0 & 0 \\
\hline Poisson's ratio & & & & & \\
\hline SUS304 & 0.3262 & 0 & $-2.002 \times 10^{-4}$ & $3.794 \times 10^{-7}$ & 0 \\
\hline $\mathrm{SI}_{3} \mathrm{~N}_{4}$ & 0.24 & 0 & 0 & 0 & 0 \\
\hline
\end{tabular}

Table 2: Convergence study of nonlinear dynamics for the FGM cylindrical shell with initial imperfection, the amplitudes of the cylindrical shell at $(x=3 L / 4, \theta=0)$ with modes $(1,1),(3,1)$ and $(5,1)$, respectively.

\begin{tabular}{||c|c|c|c|c||}
\hline$f / 10^{4} \mathrm{~N} / \mathrm{m}^{2}$ & $(N, M)$ & Amplitude $\left(10^{-3} \mathrm{~m}\right)$ & $f / 10^{4} \mathrm{~N} / \mathrm{m}^{2}$ & Amplitude $\left(10^{-3} \mathrm{~m}\right)$ \\
\hline 1 & $(1,1)$ & 2.766 & 1.6 & 2.912 \\
& $(3,1)$ & 2.640 & & 2.901 \\
& $(5,1)$ & 2.638 & & 2.897 \\
1.2 & $(1,1)$ & 2.805 & 1.8 & 3.303 \\
& $(3,1)$ & 2.727 & & 2.988 \\
& $(5,1)$ & 2.724 & & 2.983 \\
1.4 & $(1,1)$ & 2.874 & 2.0 & 3.105 \\
& $(3,1)$ & 2.814 & & 3.075 \\
& $(5,1)$ & 2.811 & & 3.069 \\
\hline
\end{tabular}

nonlinear terms, cubic nonlinear terms and external excitations are found in both of them. The difference between them is that they have different value of the coefficients and different degrees of freedom only. It is assumed that the FGM cylindrical shell is made of steel (SUS 304) and silicon nitride $\left(\mathrm{Si}_{3} \mathrm{~N}_{4}\right)$. The material parameters of the functionally graded material circular cylindrical shell are listed in Table 1, see [2]. In the following sections that the geometric of the FGM cylindrical shell are $h / R=0.002$ and $L / R=2$. Further, radial excitation is range from the 0 to $100 \mathrm{Kpa}$ and the temperature is fixed at outside surface temperature $T_{c}=400 \mathrm{~K}$ and internal surface temperature $T_{m}=300 \mathrm{~K}$. The terms of damps take the value of $\mu_{1}=\mu_{2}=\mu_{3}=697$ and the radial frequency is $\Omega_{1}=\Omega_{2}=\Omega_{3}=398 \mathrm{~Hz}$, the imperfection $h_{1}=h_{2}=0.1 h$ and $h=0.001 \mathrm{~m}$ here.

Firstly, the mode convergence is studied. The radial amplitudes of the initial imperfect cylindrical shell at $(x=3 L / 4, \theta=0)$ with modes $(1,1),(3,1)$ and $(5,1)$ are calculated in Table 2, respectively. It can be seen that the amplitude obtained with the modes $(N=3$, $M=1)$ and $(N=5, M=1)$ are nearly the same and a further increase in the number of components does not influence the results greatly. On the other hand, the value of the constant coefficients $h_{1}, h_{2}$ is only one-tenth of the thickness. From the viewpoint of convergence and qualitative analysis the first two modes can be used here. 


\subsection{Natural frequency}

Eq. (2.24) can be used to study the natural frequency of FGM circular cylindrical shell with initial imperfection. Neglecting the terms of damps, nonlinear stiffness and the forcing excitation, one can get a classical linear free-vibration equation system

$$
\{\ddot{w}\}+[M]^{-1}[K]\{w\}=[0] .
$$

Assuming the general solution of Eq. (3.1) is as

$$
\{w\}=[X] e^{\omega t}
$$

where $\omega$ is natural frequency of this shell structure. And the natural frequency can be obtained by solving the characteristic frequency equation.

Table 3 lists the first three dimensionless linear natural frequencies $\bar{\omega}=$ $\omega R \sqrt{P_{0}\left(1-v^{2}\right) / E_{0}}$ of circular cylindrical shell with and without imperfection for different volume fraction indexes, length-radius and thickness-radius ratios, respectively. It can be seen that increasing the volume fraction of the Si3N4 can make the shell more stiff and then decrease the natural frequency both for the perfect and imperfection circular cylindrical shell.

Additionally, an increase in value of $h / R$ or decline in value of $L / R$ can make the structure obtain higher bending stiffness and a higher vibration frequency. It is also shown that imperfection causes the enlarging of the natural frequency of this system compared to the circular cylindrical shell without imperfection.

Table 3: The dimensionless linear frequencies $\left(\Omega=w R \sqrt{P_{0}\left(1-v^{2}\right) / E_{0}}\right)$ of cylindrical shell with and without

\begin{tabular}{|c|c|c|c|c|c|c|}
\hline & \multicolumn{3}{|c|}{ With imperfection } & \multicolumn{3}{|c|}{ Without imperfection } \\
\hline & First order & Second order & Third order & First order & Second order & Third order \\
\hline \multicolumn{7}{|c|}{ Ceramic volume fraction $n(L / R=2, h / R=0.002)$} \\
\hline$n^{\prime}=0.5$ & 0.420583 & 1.200113 & 1.636196 & 0.415004 & 1.197866 & 1.634469 \\
\hline$n^{\prime}=1.0$ & 0.356944 & 1.045875 & 1.430839 & 0.356319 & 1.045402 & 1.430448 \\
\hline$n^{\prime}=5.0$ & 0.270211 & 0.827747 & 1.139796 & 0.269795 & 0.827365 & 1.139454 \\
\hline \multicolumn{7}{|c|}{ thickness-radius ratios $(n=0.5, L / R=2)$} \\
\hline$h / R=0.002$ & 0.416332 & 1.198667 & 1.635095 & 0.415004 & 1.197856 & 1.634440 \\
\hline$h / R=0.003$ & 0.416991 & 1.198939 & 1.635303 & 0.415171 & 1.197858 & 1.634453 \\
\hline$h / R=0.004$ & 0.420583 & 1.200113 & 1.636196 & 0.415428 & 1.197866 & 1.634469 \\
\hline \multicolumn{7}{|c|}{ length-radius ratios $(n=0.5, h / R=0.002)$} \\
\hline$L / R=1.5$ & 0.616908 & 1.347223 & 1.636196 & 0.615579 & 1.346340 & 1.635026 \\
\hline$L / R=2$ & 0.420583 & 1.200113 & 1.635298 & 0.415004 & 1.197866 & 1.634469 \\
\hline$L / R=2.5$ & 0.271499 & 1.056452 & 1.634032 & 0.271073 & 1.056079 & 1.633272 \\
\hline
\end{tabular}
imperfection for different volume fraction indexes, thickness-radius ratios and length-radius ratios.

\subsection{Nonlinear forced vibration}

The influence of radial excitation on nonlinear dynamics of the imperfect geometrically FGM cylindrical shell with different volume fraction indexes and geometry parameters 
is studied. Numerical results are obtained by using the 4th order Runge-Kutta algorithm. The bifurcation diagram, phase plane diagram and Poincare map are shown to analysis the nonlinear vibration of the geometric imperfect functionally graded material cylindrical shell. In bifurcation diagrams, the magnitude of radial excitation changes from 0 to $100 \mathrm{kPa}$.

\subsubsection{Effect of volume fraction indexes}

Nonlinear forced vibration of an initial imperfect FGM circular cylindrical shell are carried out for different volume fraction indexes $n^{\prime}=0.5, n^{\prime}=1.0$, and $n^{\prime}=5.0$. Fig. 2 depicts bifurcation diagrams of the radial excitation versus normal displacements when the radial excitation varies from 0 to $1 \times 10^{5} \mathrm{~N} / \mathrm{m}^{2}$. The horizontal axis is the parameter $f$ and the vertical axis is the value of $W_{1}, W_{2}$ and $W_{3}$. The volume fraction index of the imperfect FGM circular cylindrical shell is $n^{\prime}=0.5$. From Fig. 2 , it is seen that there is a trivial period motion for the cylindrical shell starts at $f<4.1 \times 10^{4}$. With the increasing of $f$, the trivial period motion loses its stability at $f=4.1 \times 10^{4}$ and the system enters a complex instability state of motion. For $4.1 \times 10^{4}<f<7.8 \times 10^{4}$, the FGM cylindrical shell exhibits

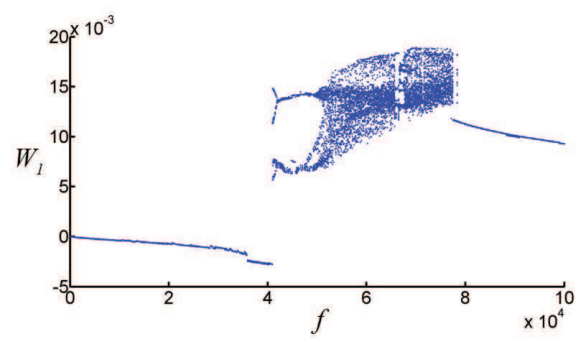

Figure 2: The bifurcation diagram of $W_{1}$ versus $f$ is given in case of $n^{\prime}=0.5, h / R=0.002, L / R=2$.
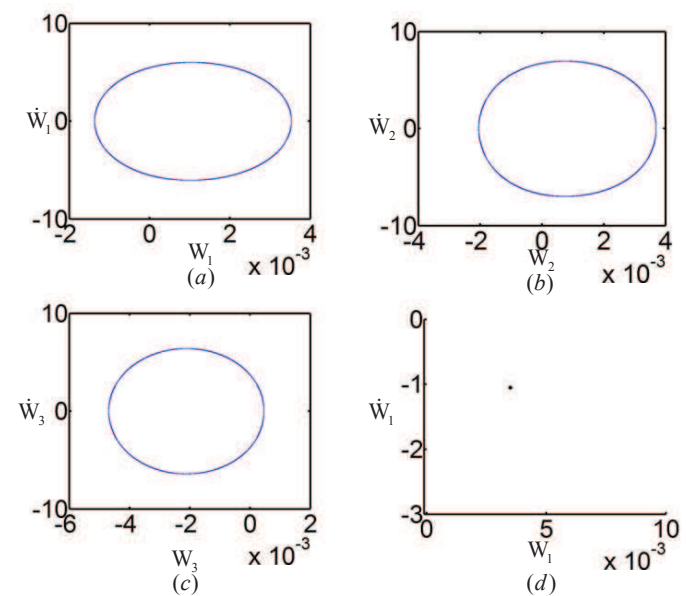

Figure 3: The period motion of the FGM cylindrical shell with the radial excitation $f=3 \times 10^{4} \mathrm{~N} / \mathrm{m}^{2}$. (a)-(c) are the phase plane diagrams on plane of the three modes respectively; $(\mathrm{d})$ the Poincare map on plane $\left(W_{1}, W_{1}\right)$. 

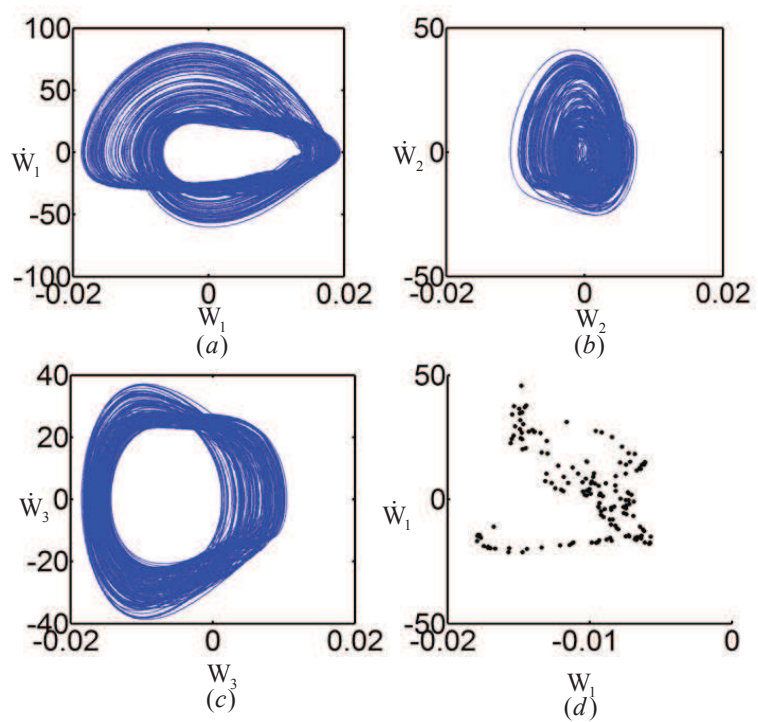

Figure 4: The chaotic motion of the FGM cylindrical shell with the radial excitation $f=6.5 \times 10^{4} \mathrm{~N} / \mathrm{m}^{2}$.
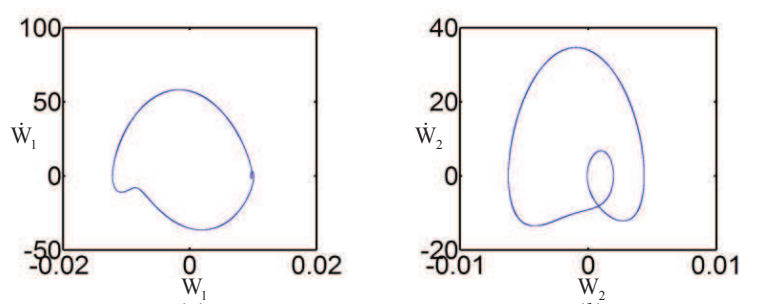

(a)
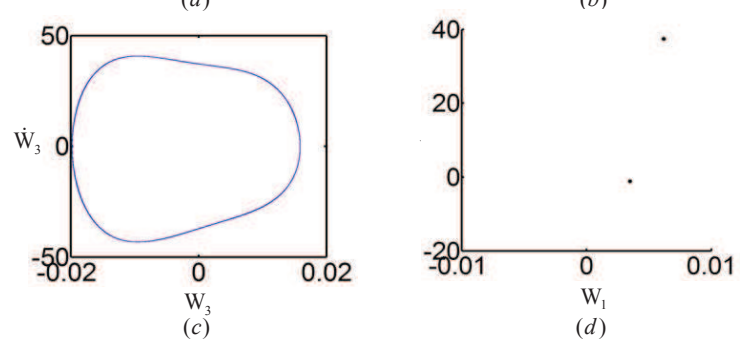

Figure 5: The period motion of the FGM cylindrical shell with the radial excitation $f=9 \times 10^{4} \mathrm{~N} / \mathrm{m}^{2}$.

chaos motion. In this chaos region there are some small period windows. The Poincare map and phase portrait are given in Figs. 3-5 to illustrate the period, chaotic and period motion of the cylindrical shell at $f=3 \times 10^{4}, f=6.5 \times 10^{4}$ and $f=9 \times 10^{4}$, respectively.

A bifurcation diagram is plotted when the volume fraction index for the ceramic is $n^{\prime}=1.0$ in Fig. 6 . As can be seen in this figure, when the radial excitation $f$ is less than $5.3 \times$ $10^{4}$ the period motion exists for the shell. A jumping behavior to another period motion 


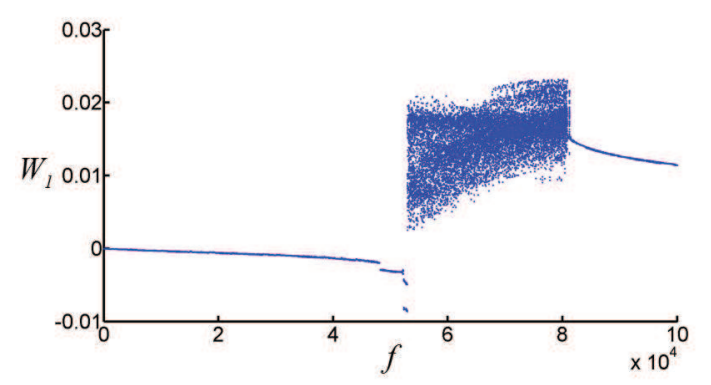

Figure 6: The bifurcation diagram of $W_{1}$ versus $f$ is given in case of $n^{\prime}=1.0, h / R=0.002, L / R=2$.
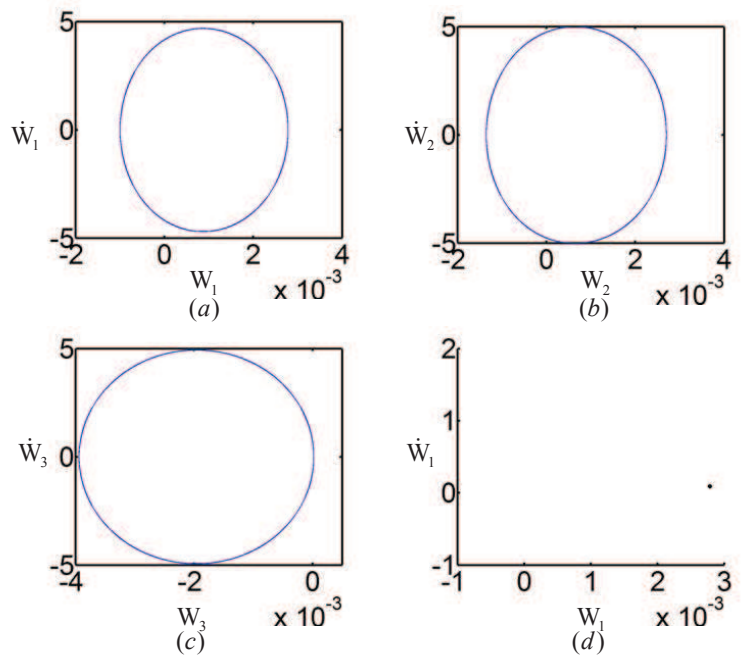

Figure 7: The period motion of the FGM cylindrical shell with the radial excitation $f=3 \times 10^{4} \mathrm{~N} / \mathrm{m}^{2}$.

occurs at $f=8.1 \times 10^{4}$. After the critical point, as the radial excitation is increased up to a chaotic region with two tiny periodic windows appears by transcritical bifurcation. Figs. 7-9 show the motion of shell at radial excitation of $f=3 \times 10^{4}, f=6.5 \times 10^{4}$ and $f=9 \times 10^{4}$, respectively.

Fig. 10 is the bifurcation diagrams of the imperfect FGM cylindrical shell with $n^{\prime}=5.0$. As observed in Fig. 10, there is a trivial period motion in the beginning until radial excitation is increased up to $8.1 \times 10^{4}$, where a jumping behavior is detected. The system jump to a multi-period motion and then at $f=8.5 \times 10^{4}$ the multi-period motion becomes the chaos. But just after a very small increase in excitation a short period motion is observed and then the system enters a chaotic region. Figs. 9-11 present the motion of shell at radial excitation of $f=3 \times 10^{4}, f=8.1 \times 10^{4}$ and $f=9 \times 10^{4}$, respectively. It can be shown from Figs. 11-13 that the amplitude of the chaotic motion is larger than one of the periodic motion.

Comparison of Figs. 2, 6 and 10 reveals that generally they have the similar dynamic 

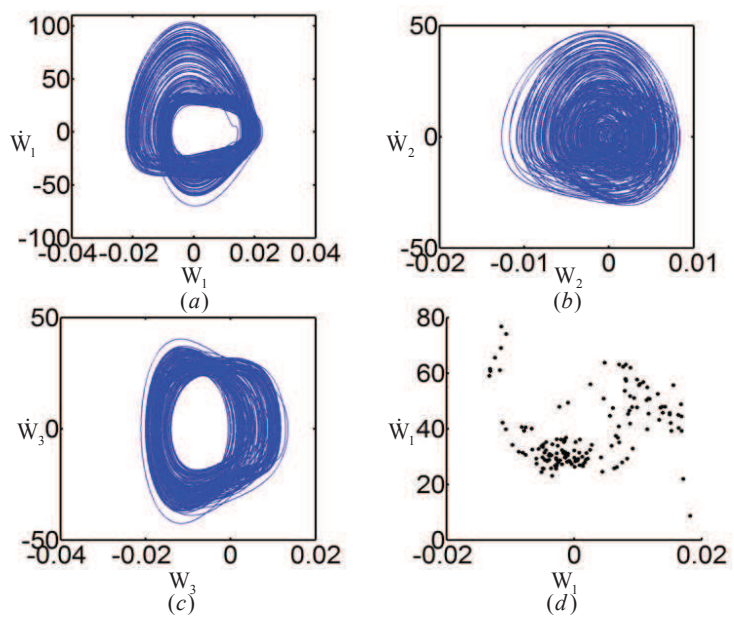

Figure 8: The chaotic motion of the FGM cylindrical shell with the radial excitation $f=6.5 \times 10^{4} \mathrm{~N} / \mathrm{m}^{2}$.
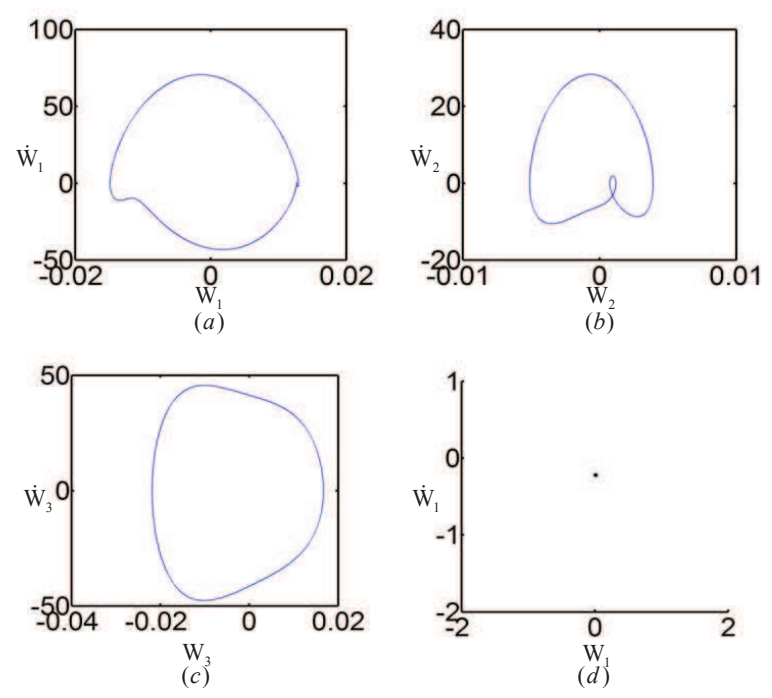

Figure 9: The period motion of the FGM cylindrical shell with the radial excitation $f=9 \times 10^{4} \mathrm{~N} / \mathrm{m}^{2}$.

behaviors but the periodic and chaotic regions are different. Chaos occur need larger excitation when the value of volume fraction indexes increases. When the forcing excitation magnitude is approximately $f=4.1 \times 10^{4}, f=5.3 \times 10^{4}$, and $f=8.7 \times 10^{4}$, the FGM circular cylindrical shell tends to undergo the changes from periodic to chaotic motions for the case that $n^{\prime}$ is as $0.5,1.0$ and 5.0, respectively.

\subsubsection{Effect of initial geometric imperfection}

Influences of the initial geometric imperfections on nonlinear dynamical responses of FGM circular cylindrical shell are researched. First of all, in Eq. (2.9) if $h_{1}$ and $h_{2}$ are all 


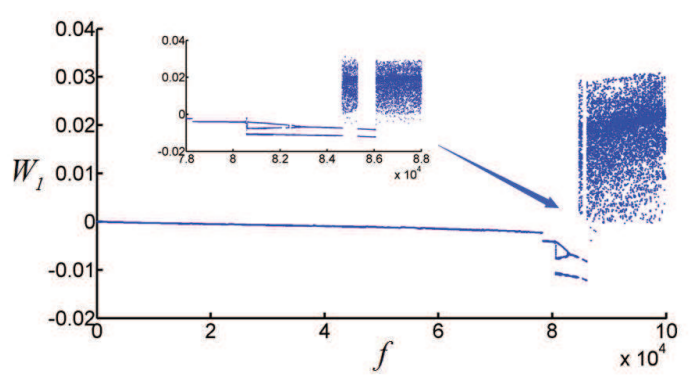

Figure 10: The bifurcation diagram of $W_{1}$ versus $f$ is given in case of $n^{\prime}=5.0, h / R=0.002, L / R=2$.
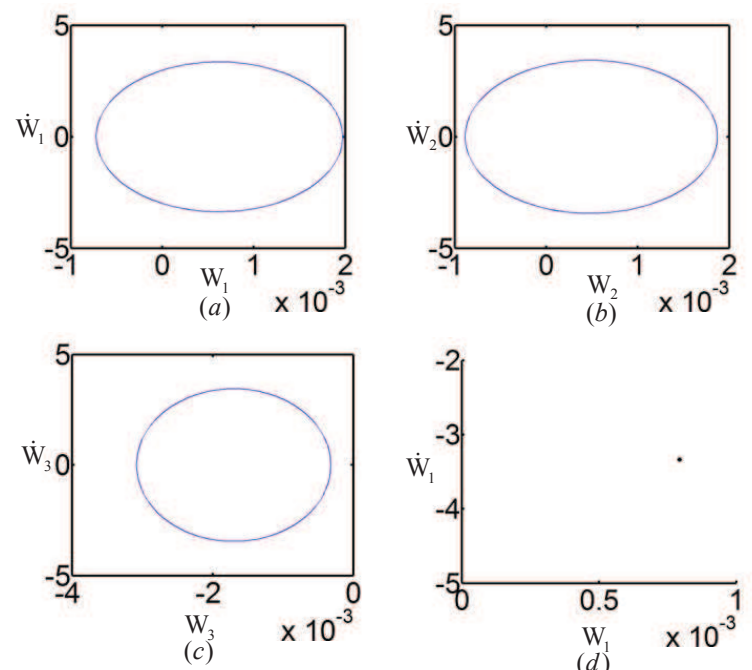

Figure 11: The period motion of the FGM cylindrical shell with the radial excitation $f=3 \times 10^{4} \mathrm{~N} / \mathrm{m}^{2}$.

zero this circular cylindrical shell is perfect. The bifurcation diagram shown in Fig. 14 is plotted for the same data used in Fig. 2. It is seen that for the perfect circular cylindrical shell only periodic motion over the range of $f \in\left(0,1 \times 10^{5}\right)$ is detected. The Poincare map and phase portrait are given in Fig. 15 to illustrate the period motion of the system when the excitation is as $f=4 \times 10^{4}$. The bifurcation diagram shown in Fig. 16 is plotted for $h_{1}=0$ and $h_{2}=0.1 h$. It means that the shell presents the imperfection of axisymmetric hyperbolic functions as

$$
w^{*}=h_{2}\left(\cosh \frac{\lambda_{m} x}{L}-\cos \frac{\lambda_{m} x}{L}-\sigma_{m}\left(\sinh \frac{\lambda_{m} x}{L}-\sin \frac{\lambda_{m} x}{L}\right)\right) .
$$

From Fig. 16, it is seen that there is a trivial amplitude period motion for the cylindrical shell until the excitation is at $3.5 \times 10^{4}$. With the increasing of excitation $f$, the trivial period motion loses its stability at $f=3.5 \times 10^{4}$ and the system enters a complex instability state of motion. For $3.5 \times 10^{4}<f<4 \times 10^{4}$, the FGM cylindrical shell exhibits chaos motion. 


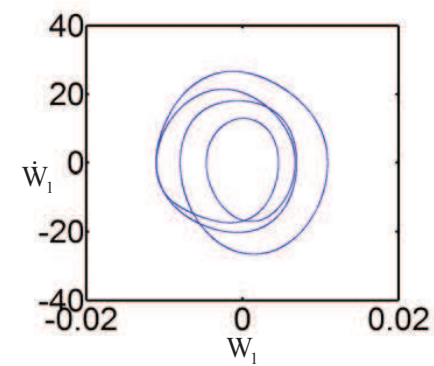

(a)

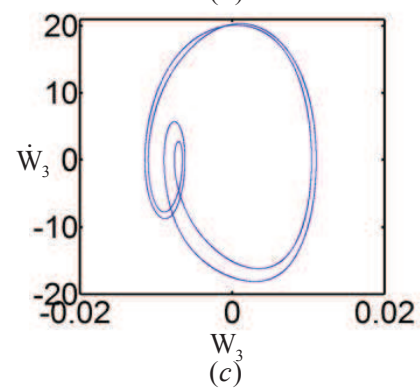

$(c)^{3}$

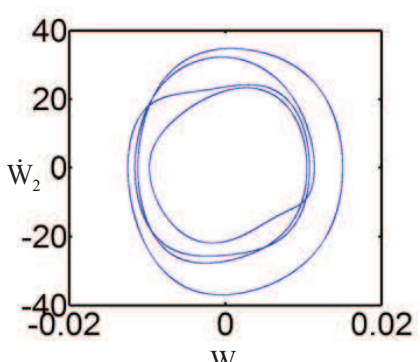

(b)

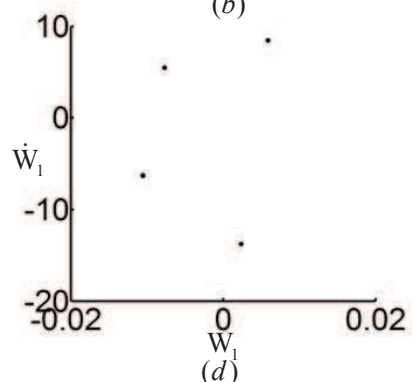

$(d)$

Figure 12: The multi-period motion of the FGM cylindrical shell with the radial excitation $f=8.1 \times 10^{4} \mathrm{~N} / \mathrm{m}^{2}$.

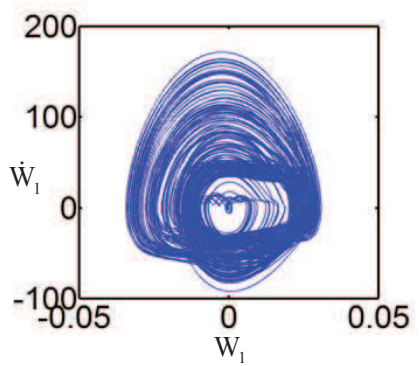

(a)

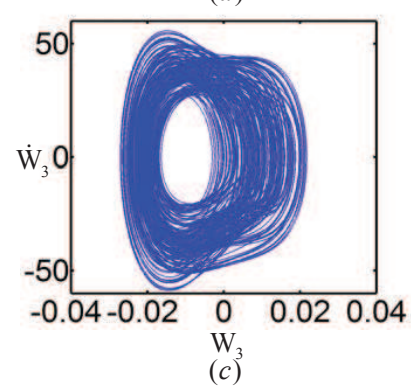

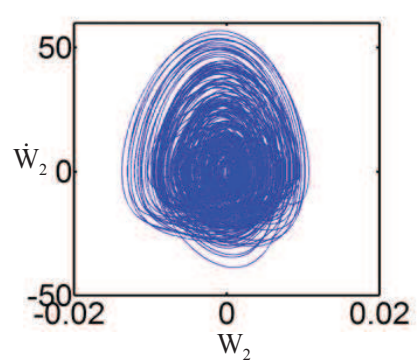

(b)

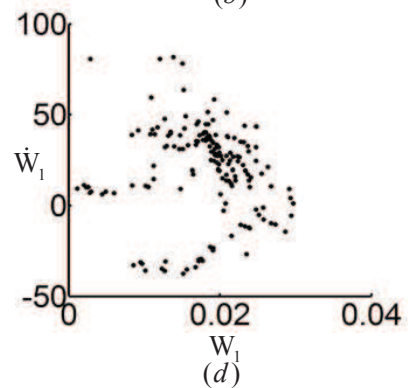

Figure 13: The chaotic motion of the FGM cylindrical shell with the radial excitation $f=9 \times 10^{4} \mathrm{~N} / \mathrm{m}^{2}$.

Then it enters a multi-period motion range from $f=4 \times 10^{4}$ to $5.1 \times 10^{4}$. But for the case of $f \geq 5.1 \times 10^{4}$, the system enters a chaos motion again. The Poincare map and phase portrait are given Figs. 17-20 to illustrate the period, chaotic and period motion of the 


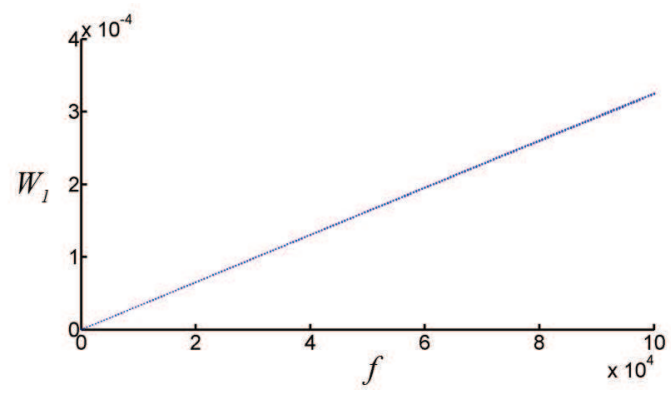

Figure 14: The bifurcation diagram of $W_{1}$ versus $f$ is given when the shell is perfect, and other parameters are $n^{\prime}=0.5, h / R=0.002, L / R=2$, respectively.
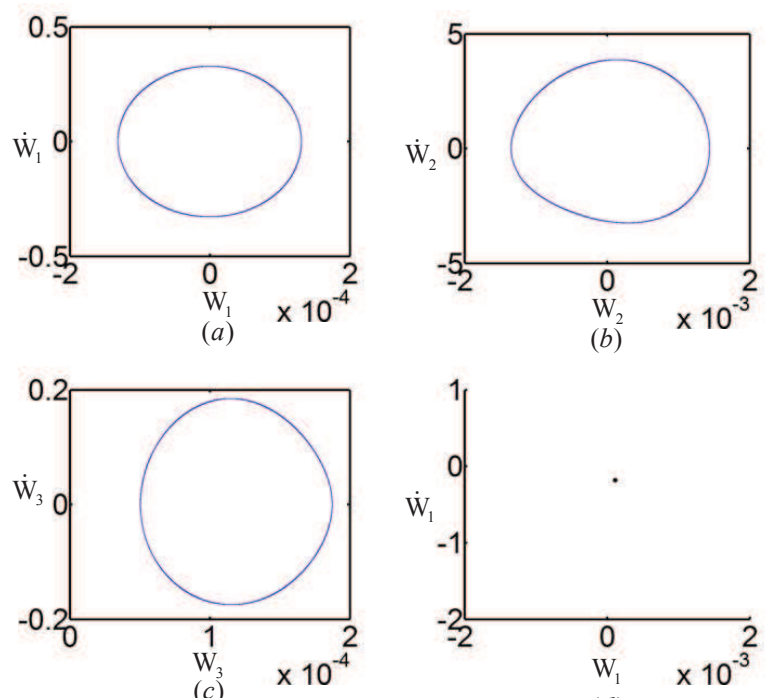

(d)

Figure 15: The period motion of the FGM cylindrical shell with the radial excitation $f=4 \times 10^{4} \mathrm{~N} / \mathrm{m}^{2}$.

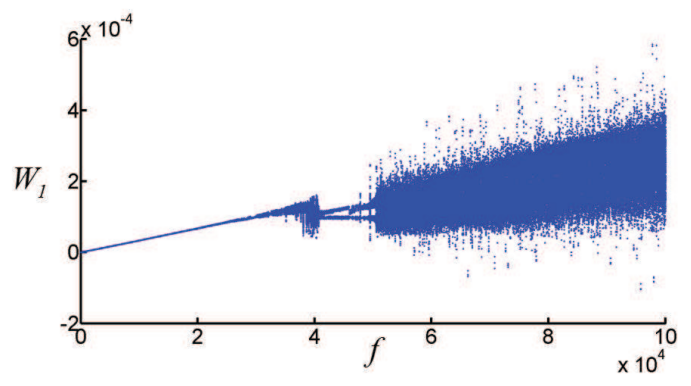

Figure 16: The bifurcation diagram of $W_{1}$ versus $f$ is given when the coefficients take the value of $h_{1}=0$ and $h_{2}=0.1 \mathrm{~h}$ in Eq. (2.9), and other parameters are $n^{\prime}=0.5, h / R=0.002, L / R=2$, respectively. 


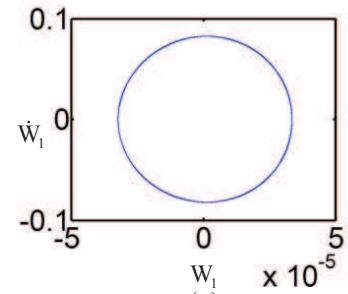

(a)

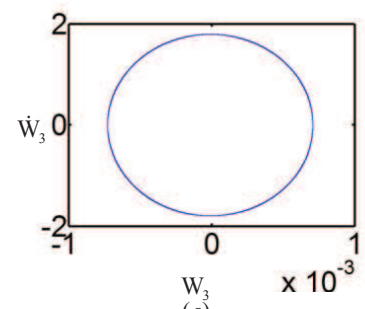

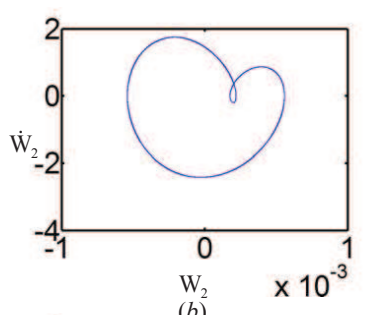

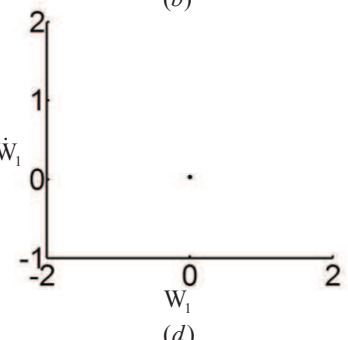

Figure 17: The period motion of the FGM cylindrical shell with the radial excitation $f=1 \times 10^{4} \mathrm{~N} / \mathrm{m}^{2}$.
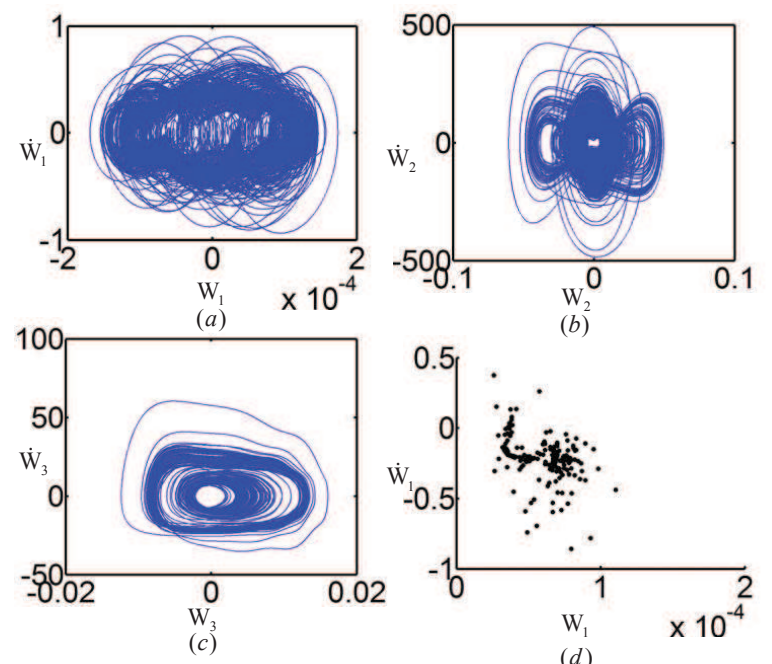

Figure 18: The chaotic motion of the FGM cylindrical shell with the radial excitation $f=4 \times 10^{4} \mathrm{~N} / \mathrm{m}^{2}$.

cylindrical shell at $f=1 \times 10^{4}, f=4 \times 10^{4}$, and $f=6 \times 10^{4}$, respectively.

\section{Conclusions}

The dynamic responses of clamped-clamped FGM cylindrical shell at two edges with small initial geometric imperfection under complex loads are studied. The effects of radial harmonic excitation combined with thermal loads are considered. The small initial 

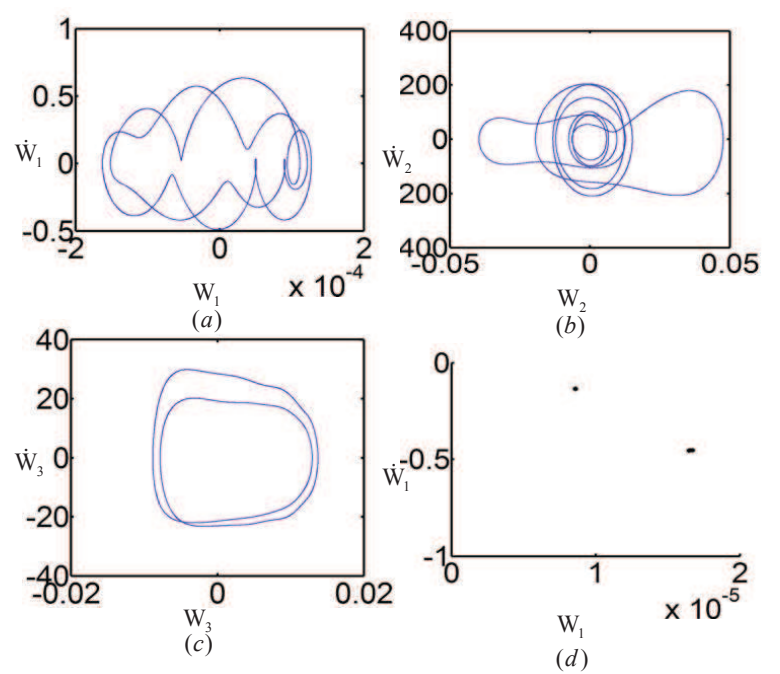

Figure 19: The multi-period motion of the FGM cylindrical shell with the radial excitation $f=4.5 \times 10^{4} \mathrm{~N} / \mathrm{m}^{2}$.
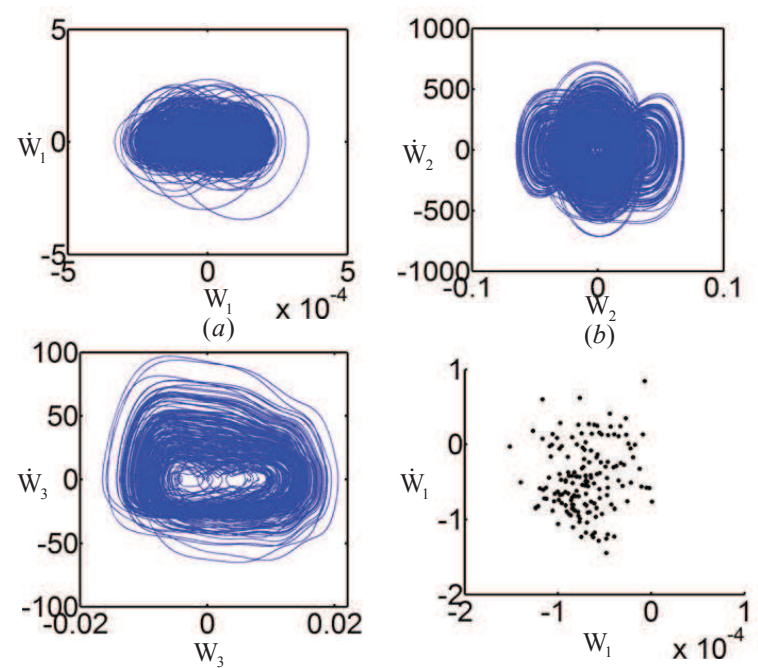

(c)

$(d)$

Figure 20: The chaotic motion of the FGM cylindrical shell with the radial excitation $f=6 \times 10^{4} \mathrm{~N} / \mathrm{m}^{2}$.

geometric imperfection of the cylindrical shell is characterized with the similar shape as radial mode. The effective material properties vary in the radial direction following a power law in terms of the volume fractions. According to the classical shell theory and von Karman type geometric nonlinear relation, the nonlinear partial differential equation is deduced by the Hamiltons principle. The nonlinear dynamic equations are truncated by the Galerkin technique, using the modal expansion with the inclusion of axisymmetric and asymmetric modes. The effects of volume fraction indexes, length-radius and 
thickness-radius ratios on natural frequencies of the cylindrical shell with imperfection and without imperfection are given.

It can be seen that increasing the volume fraction of silicon nitride can decrease the natural frequency of either for the perfect circular cylindrical shell or the imperfection circular cylindrical shell. Increasing the value of $h / R$ (and an equivalent decline in value of $L / R$ ) may result in higher shell bending stiffness and a higher vibration frequency. It is also shown that imperfection causes the enlarging of the natural frequency of this system compared to the perfect circular cylindrical shell.

Numerical results are presented by bifurcation diagrams, phase plane diagrams and Poincare maps. It is shown that under the certain conditions the shell has the similar dynamic behaviors but the periodic and chaotic regions are different for different volume fractions. With the increasing of ceramics, much more excitation is needed for chaotic motions of the system. It also shows that nonlinear forced vibration is sensitive to the shape of imperfections.

\section{Acknowledgements}

The authors acknowledge the financial support of National Natural Science Foundation of China through grant Nos. 11472056 and 11272063, Natural Science Foundation of Tianjin City through grant No. 13JCQNJC04400.

\section{References}

[1] H. Haddadpour, H. M. Navazi And F. Shadmehri, Nonlinear oscillations of a fluttering functionally graded plate, Compos. Struct., 79 (2007), pp. 242-250.

[2] C. T. LOY, K. Y. LAM AND J. N. REDDY, Vibration of functionally graded cylindrical shells, Int. J. Mech. Sci., 41 (1999), pp. 309-324.

[3] S. C. Pradhan, C. T. LOY, K. Y. LAM AND J. N. RedDY, Vibration characteristics of functionally graded cylindrical shell under various boundary conditions, Appl. Acoust., 61 (2000), pp. $111-129$.

[4] S. H. ARShAD, M. N. NAEEM AND N. SultanA, Frequency analysis of functionally graded material cylindrical shells with various volume faction laws, Proc. Inst. Mech. Eng. C, J. Mech. Eng. Sci., 221 (2007), pp. 1483-1495.

[5] A. G. SHAH, T. MAHMOOD AND M. N. NAEEM, Vibrations of FGM thin cylindrical shells with exponential volume fraction law, Appl. Math. Mech., 30 (2009), pp. 607-616.

[6] M. AsGARI AND M. AKHLAGHI, Natural frequency analysis of 2D-FGM thick hollow cylinder based on three-dimensional elasticity equations, Euro. J. Mech. A Solids, 30 (2011), pp. 72-81.

[7] R. K. BHANGALE AND N. GANESAN, Free vibration studies of simply supported nonhomogeneous functionally graded magneto-electro-elastic finite cylindrical shells, J. Sound Vib, 288 (2005), pp. $412-422$.

[8] Z. Y. CAO AND H. N. WANG, Free vibration of FGM cylindrical shells with holes under various boundary conditions, J. Sound Vib, 306 (2007), pp. 227-237. 
[9] H. A. Sepiani, A. Rastgoo, F. Ebrahimi AND A. A. GHORBAnPour, Vibration and buckling analysis of two-layered functionally graded cylindrical shell, considering the effects of radial shear and rotary inertia, Mater. Des., 31 (2010), pp. 1063-1069.

[10] A. BAHTUI AND M. R. EsLAmI, Generalized coupled thermoelasticity of functionally graded cylindrical shells, Int. J. Non-Linear Mech., 69 (2007), pp. 676-697.

[11] T. Y. NG, K. Y. LAM, K. M. LIEW AND J. N. REDDY, Dynamic stability analysis of functionally graded cylindrical shell under periodic axial loading, Int. J. Solids Struct., 38 (2001), pp. 12951309.

[12] S. Mahmoudkhani, H. M. Mavazi And H. Haddadpour, An analytical study of the non-linear vibrations of cylindrical shells, Int. J. Non-Linear Mech., 46 (2011), pp. 1361-1372.

[13] D. H. BICH AND N. X. NGUYEN, Nonlinear vibration of functionally circular cylindrical shells based on improved Donnell equations, J. Sound Vib, 331 (2012), pp. 5488-5501.

[14] Y. X. HAO, W. ZHANG AND J. YANG, Nonlinear dynamics of cantilever FGM cylindrical shell under 1:2 internal resonance relations, Mech. Adv. Mater. Struct., 20 (2013), pp. 819-833.

[15] C. C. DU AND Y. H. LI, Nonlinear resonance behavior of functionally graded cylindrical shells in thermal environments, Compos. Struct., 102 (2013), pp. 164-174.

[16] H. S. SHEN, Nonlinear vibration of shear deformable FGM cylindrical shells surrounded by an elastic medium, Compos. Struct., 94 (2012), pp. 1144-1154.

[17] M. StRozzi AND F. Pellicano, Nonlinear vibrations of functionally graded cylindrical shells, Thin-Walled Struct., 67 (2013), pp. 63-77.

[18] G. G. SHENG AND X. WANG, Nonlinear vibration control of functionally graded laminated cylindrical hells, Compos. B Eng., 52 (2013), pp. 1-10.

[19] G. G. SHENG, X. WANG G. FU AND H. HU, The nonlinear vibrations of functionally graded cylindrical shells surrounded by an elastic foundation, Nonlinear Dyn., 78 (2014), pp. 1421-1434.

[20] R. KADOLI AND N. GANESAN, Buckling and free vibration analysis of functionally graded cylindrical shells subjected to a temperature-specified boundary condition, J. Sound Vib., 289 (2006), pp. 450-480.

[21] W. ZHANG, Y. X. HAO AND J. YANG, Nonlinear dynamics of FGM circular cylindrical shell with clampedclamped edges, Compos. Struct., 94 (2012), pp. 1075-1086.

[22] F. Alijani AND M. AmABILI, Non-linear vibrations of shells: A literature review from 2003 to 2013, Int. J. Non-Linear Mech., 58 (2014), pp. 233-257.

[23] M. Rafiee, M. Mohammadi, B. S. Aragh and H. Yaghoobi, Nonlinear free and forced thermo-electro-aero-elastic vibration and dynamic response of piezo-electric functionally graded laminated composite shells, Part I: theory and analytical solutions, Compos. Struct., 103 (2013), pp. 179-187.

[24] H. S. SHEN, Postbuckling of axially loaded FGM hybrid cylindrical shells in thermal environments, Compos. Sci. Technol., 65 (2005), pp. 1675-1690.

[25] M. SHARIYAT, Dynamic thermal buckling of suddenly heated temperature dependent FGM cylindrical shells, under combined axial compression and external pressure, Int. J. Solids Struct., 45 (2008), pp. 2598-2612.

[26] H. W. HUANG AND Q. HAN, Buckling of imperfect functionally graded cylindrical shells under axial compression, Euro. J. Mech. A Solids, 27 (2008), pp. 1026-1036.

[27] M. SHARIYAT, M. NIKKHAH AND R. KAZEMI, Exact and numerical elastodynamic solutions for thick-walled functionally graded cylinders subjected to pressure shocks, Int. J. Pressure Vessels Piping, 88 (2011), pp. 75-87.

[28] S. M. Hosseini, J. SLADEK AND V. SLADEK, Meshless local Petrov-Galerkin method for coupled thermoelasticity analysis of a functionally graded thick hollow cylinder, Eng. Anal. Bound. Elem., 
35 (2011), pp. 827-835.

[29] M. SHARIYAT, Nonlinear transient stress and wave propagation analyses of the FGM thick cylinders, employing a unified generalized thermoelasticity theory, Int. J. Mech. Sci., 65 (2012), pp. 24-37.

[30] N. D. DUC AND T. Q. QUAN, Nonlinear response of imperfect eccentrically stiffened FGM cylindrical panels on elastic foundation subjected to mechanical loads, Euro. J. Mech. A Solids, 46 (2014), pp. $60-71$.

[31] W. P. Hu, Z. C. Deng, S. M. HAN AND W. ZHANG, Generalized multi-symplectic integrators for a class of Hamiltonian nonlinear wave PDEs, J Comput Phys., 235 (2013), pp. 394-406.

[32] W. P. HU, Q. J. LI, X. H. JIANG AND Z. C. DENG, Coupling dynamic behaviors of spatial flexible beam with weak damping, Int. J. Numer. Meth. Eng, DOI: 10.1002/nme.5477.

[33] W. P. Hu, Z. C. DENG, B. WANG, H. JIANG AND O. YANG, Chaos in an embedded single-walled carbon nanotube, Nonlinear Dyn., 72 (2013), pp. 389-398.

[34] W. P. Hu AND Z. C. DENG, Chaos in embedded fluid-conveying single-walled carbon nanotube under transverse harmonic load series, Nonlinear Dyn., 79 (2015), pp. 325-333.

[35] J. N. REDDY, Mechanics of Laminated Composite Plates and Shells: Theory and Analysis, CRC Press, New York, 2004.

[36] M. Rouguia, F. Moussaouib And R. Benamar, Geometrically non-linear free and forced vibrations of simply supported circular cylindrical shells:Asemi-analytical approach, Int. J. NonLinear Mech., 42 (2007), pp. 1102-1115.

[37] M. Amabili, F. Pellicano AND M. P. Paidoussis, Non-linear dynamic and stability of circular cylindrical shells containing flowing fluid, Part II: Large-amplitude vibration without flow, J. Sound Vib., 228 (1999), pp. 1103-1124.

[38] S. C. Pradhana, C. T. LOYA, K. Y. LAma AND J. N. RedDy, Vibration characteristics of functionally graded cylindrical shells under various boundary conditions, Appl. Acoust., 61 (2000), pp. 111-129.

[39] H. S. SHEN, Postbuckling of shear deformable FGM cylindrical shells surrounded by an elastic medium, Int. J. Mech. Sci., 51 (2009), pp. 372-383.

[40] K. N. Karagiozis, M. P. Paidoussis, M. Amabili AND A. K. MisRa, Nonlinear stability of cylindrical shells subjected to axial flow: Theory and experiments, J. Sound Vib., 309 (2008), pp. 637-676.

[41] P. B. GONCALVES AND Z. G. DELPRADO, Effect of non-linear modal interaction on the dynamic instability of axially excited cylindrical shells, Compos. Struct., 82 (2004), pp. 2621-2634.

[42] M. AmabiLI, F. PelliCANO AND M. P. PAIDOUSSIS, Non-linear vibrations of simply supported circular cylindrical shells coupled to quiescent fluid, J. Fluids Struct., 12 (1998), pp. 883-918.

[43] M. Amabili, F. Pellicano AND M. P. Paidoussis, Nonlinear dynamics and stability of circular cylindrical shells containing flowing fluid, Part I: Stability, J. Sound Vib., 225 (1999), pp. 655-699.

[44] C. C. DU, Y. H. LI AND X. S. JIN, Nonlinear forced vibration of functionally graded cylindrical thin shells, Thin-Walled Struct., 78 (2014), pp. 26-36.

[45] M. AmABILI, Nonlinear Vibrations and Stability of Shells and Plates, New York, Cambridge University Press, 2008.

[46] A. NOSIR AND J. N. REDDY, A study of non-linear dynamic equations of higher-order deformation plate theories, Int. J. Non-Linear Mech., 26 (1991), pp. 233-249.

[47] A. BHIMARADDI, Large amplitude vibrations of imperfect antisymmetric angle-ply laminated plates, J. Sound Vib., 162 (1999), pp. 457-470.

[48] M. DARABI, M. DARVIZeH AND A. DARVIZeH, Non-linear analysis of dynamic stability for 
functionally graded cylindrical shells under periodic axial loading, Compos. Struct., 83 (2008), pp. 201-211.

[49] J. YANG, Y. X. HAO, W. ZHANG AND S. KitIPORNCHAI, Nonlinear dynamic response of a functionally graded plate with a through-width surface crack, Nonlinear Dyn., 59 (2010), pp. 207219. 